\title{
The Ramsey method in high-precision mass spectrometry with Penning traps: Theoretical foundations
}

\author{
Martin Kretzschmar \\ Institut für Physik, Johannes-Gutenberg-Universität, 55099 Mainz, Germany \\ Received 23 December 2006; received in revised form 2 April 2007; accepted 2 April 2007 \\ Available online 6 April 2007
}

\begin{abstract}
This paper presents in a quantum mechanical framework a theoretical description of the interconversion of the magnetron and modified cyclotron motional modes of ions in a Penning trap due to excitation by external rf-quadrupole fields with a frequency near the true cyclotron frequency. The work aims at a correct description of the resonance line shapes that are observed in connection with more complicated excitation schemes using several excitation pulses, such as Ramsey's method of separated oscillating fields. Quantum mechanical arguments together with the "rotating wave approximation" suggest a model Hamiltonian that permits a rigorous solution of the corresponding Heisenberg equations of motion. We show that the ion motion in a Penning trap with an additional rf-quadrupole field is dynamically analogous to nuclear-magnetic-resonance. This is done by introducing the concept of the "Bloch vector operator", which is a vector operator obeying the commutation rules of an angular momentum operator and which is the analogue of a nuclear spin. During the quadrupole excitation the expectation value of the Bloch vector operator, which is an ordinary real three-vector, performs a precessional and nutational motion similar to the spin in nuclear-magnetic-resonance experiments. The frequency of the interconversion of the magnetron and modified cyclotron motional modes of the ions is the analogue of the Rabi frequency. Hence the applicability of Ramsey's ideas to Penning trap physics becomes understandable. Resonance line shapes are deduced from the general solution of the dynamical problem for arbitrary values of the excitation time, pulse structure and detuning of the quadrupole radiation. Results are given for excitation schemes with up to five pulses. A comparison of the theoretical results to experimental data is found in the accompanying paper by George et al. [S. George, et al., IJMS, this issue].
\end{abstract}

(C) 2007 Elsevier B.V. All rights reserved.

PACS: $07.75 .+\mathrm{h} ; 21.10 . \mathrm{Dr} ; 32.10 . \mathrm{Bi} ; 82.80 . \mathrm{Qx}$

Keywords: Penning trap; Ion motion; Mass spectrometry; Ramsey excitation

\section{Introduction}

In 1949, N.F. Ramsey proposed a new "molecular beam resonance method with separated oscillating fields" [2,3]. It proved to be extremely successful, leading to higher accuracy and new types of experiments, it was recognized by the 1989 Nobel prize. For a review of the method the reader is referred to Ramsey's Nobel lecture [4]. The essence of the method can be easily explained by the example of magnetic-resonance experiments on nuclear spins. In these experiments one acts on the nuclear magnetic moment with a strong, homogeneous and static magnetic field $\mathbf{B}_{0}$ and another weak, homogeneous, and oscillating magnetic field $\mathbf{B}_{1}$ with a frequency $\omega$ equal or close to the Larmor frequency $\omega_{\mathrm{L}}$. The particle beam traverses the spatial region where these fields are located. In Rabi type experiments $\mathbf{B}_{0}$ and $\mathbf{B}_{1}$ are present in the same spatial volume, in Ramsey type experiments the volume is divided into three subregions, an entrance and an exit region, where the weak oscillating field $\mathbf{B}_{1}$ is acting in addition to $\mathbf{B}_{0}$, and a central region, where the weak oscillating field is absent and where the nuclear moment feels only the the strong static field $\mathbf{B}_{0}$ and eventually other fields. It is essential that the oscillating fields in the entrance and exit regions have a precisely defined phase relation.

E-mail address: Martin.Kretzschmar@uni-mainz.de. 
In 1992, Bollen et al. [5] suggested to use the Ramsey technique to improve the accuracy of mass determinations by means of Penning trap mass spectrometry. First experiments have been performed by Bollen et al. with the ISOLTRAP installation at CERN [5] and later on by Bergström et al. at the SMILETRAP installation in Stockholm [6]. After a series of preparatory steps an ion sample trapped in a hyperbolic precision trap in a state of pure magnetron motion is acted upon by a pulse of quadrupole rf-radiation with a frequency near the true cyclotron frequency, and with well-defined duration and amplitude. The motional state of the ion sample is transformed resonantly into a state of modified cyclotron motion [15], which is then detected by a time-of-flight technique [7]. The resonance peak occurs at the true cyclotron frequency, which is inversely proportional to the ion mass that one wants to measure. Thus the measurement of the resonance frequency implies the desired mass determination. The accuracy of the measurement is determined by the width and shape of the resonance line. Ramsey techniques are expected to yield more narrow linewidths and more accurate results. In Ramsey's experiments the molecular beams traversed separate spatial regions where the oscillating magnetic fields were present, in our case the ions are confined all the time to the same spatial location, the trap interior, the pulses of quadrupole rf-radiation are applied at two or more time periods separated by waiting times. The experiments by Bollen et al. [5] had shown that a qualitative understanding of the observed line shapes could be obtained by simply considering the Fourier transform of the applied pulse sequence. But it was also realized that the exact line shape, in particular that of the most important central resonance peak, could only be understood on the basis of a more elaborate theoretical analysis. Because of this uncertainty about the theoretical foundations of the method the experimental implementation of these ideas was delayed until recently [1].

The purpose of this paper is to put the application of the Ramsey technique in Penning trap mass spectrometry on a solid theoretical foundation. On the fundamental level we wish to understand the relation between Ramsey's experiments on nuclearmagnetic-resonance and our problem of ion motion in the Penning trap. We introduce the concept of the Bloch vector operator [8], which incorporates the physics of interconversion of the motional modes. The operator components obey angular momentum commutation rules and thus permit to establish a close parallelism between both problems. The expectation value of this operator, being an ordinary three-vector, performs similar precessional and nutational motions as the nuclear spin in Ramsey's case. The threecomponent of this vector indicates the degree of conversion of magnetron into modified cyclotron motion that has been achieved at a given time. Thus analyzing the motion of the Bloch vector as function of the pulse structure of the quadrupole radiation, i.e., of the number, duration and amplitudes of the pulses and of the duration of the waiting periods, we can derive all desired information on the line shape and structure of the resonance signals, which can then be compared to measurement results after the non-linear response of the time-of-flight detection technique [7] has been taken into account.

The plan of the paper is as follows: we begin with a short review of the theory of ion motion in the ideal Penning trap, from the classical and the quantum viewpoint, emphasizing that the Hamiltonian is a composite of two ordinary harmonic oscillators for axial and modified cyclotron modes and one inverted harmonic oscillator for the magnetron mode. Then the addition of an electric rf-quadrupole field is analyzed. Its interaction with the trapped ions is simplified by the rotating wave approximation and by dropping terms that are not relevant for driving frequencies near the true cyclotron frequency. Thus a model interaction emerges that describes the interconversion of the magnetron and modified cyclotron modes as due to the absorption (emission) of one photon of the quadrupole field with simultaneous creation (annihilation) of one quantum of the modified cyclotron oscillator and annihilation (creation) of one quantum of the magnetron oscillator. The Heisenberg equations of motion for the creation and annihilation operators are linear and can be exactly solved. Classical ion trajectories are obtained in the quantized approach as expectation values for minimum uncertainty coherent states. Thus we can provide explicite analytical expressions describing the motion of an ion under the influence of the quadrupole field. Next the Bloch vector operator is introduced, its equations of motion are solved rigorously. It is found that the dynamics of the Bloch vector operator in Penning trap physics and that of the nuclear spin in the realm of nuclear-magneticresonance are physical realizations of the same abstract dynamical system. Two different, but equivalent methods for calculating the resonance line shapes for the conversion of magnetron into modified cyclotron motion are presented. The Ramsey method is explored in detail for two-, three-, four-, and five-pulse structures, also asymmetric and phaseshifted structures are considered.

\section{Theoretical description of the ion motion}

For an overview of the theoretical description of physics with Penning traps we refer the reader to reviews $[9,10]$ and monographs $[11,12]$ in the literature. In this paper we restrict ourselves to fundamentals needed to establish our notation and to the further developments required for the theoretical analysis of the experimentally observed line shapes.

\subsection{Ion motion in the ideal Penning trap}

The ideal Penning trap is defined by its electromagnetic field configuration, namely a superposition of an homogeneous magnetic field $\mathbf{B}=B_{0} \mathbf{e}_{3}$ in the "axial" direction" ${ }^{1}$ with an electrostatic quadrupole field $\mathbf{E}=-\nabla \Phi_{0}$ corresponding to

${ }^{1}$ This choice conforms to that in Refs. [8,13,14]. Comparing our formulas to those of Brown and Gabrielse [9] one must bear in mind their choice $\mathbf{B}=-B_{0} \mathbf{e}_{3}$. 
the potential

$$
\Phi_{0}(x, y, z)=\frac{U}{2 z_{0}^{2}+r_{0}^{2}}\left(2 z^{2}-x^{2}-y^{2}\right),
$$

where $U$ is the potential difference between the two hyperboloidal equipotential surfaces $2 z^{2}-x^{2}-y^{2}=-r_{0}^{2}$ (ring electrode) and $2 z^{2}-x^{2}-y^{2}=2 z_{0}^{2}$ (endcap electrodes). As shown in greater detail in Refs. [13,14] the motion of an ion with mass $m$ and electric charge $q$ in this field configuration can be analyzed in terms of cartesian coordinates and velocities, $x, y, z$ and $\dot{x}, \dot{y}, \dot{z}$, respectively, using Newtonian or Lagrangian equations. The magnetic field and the charge-to-mass ratio define the "true" cyclotron frequency $\omega_{\mathrm{c}}=q B_{0} / m$, the electric potential defines the axial frequency $\omega_{z}=\sqrt{4 q U / m\left(2 z_{0}^{2}+r_{0}^{2}\right)}$. Most advantageous is the Hamiltonian formalism, in terms of $x, y, z$ and the canonical momenta

$$
p_{x}=m \dot{x}-\frac{1}{2} m \omega_{\mathrm{c}} y, \quad p_{y}=m \dot{y}+\frac{1}{2} m \omega_{\mathrm{c}} x, \quad p_{z}=m \dot{z},
$$

because after a suitable canonical transformation to modal coordinates $q_{+}, q_{-}, q_{3}$ and the corresponding canonical momenta $p_{+}, p_{-}, p_{3}$ the dynamical system is resolved into three uncoupled harmonic oscillators, one of them an inverted oscillator, with the characteristic frequencies $\omega_{+}$(modified cyclotron frequency), $\omega_{-}$(magnetron frequency), and $\omega_{z}$ (axial frequency), where

$$
\omega_{ \pm}=\frac{1}{2}\left(\omega_{\mathrm{c}} \pm \omega_{1}\right) \quad \text { with } \omega_{1}=\sqrt{\omega_{\mathrm{c}}^{2}-2 \omega_{z}^{2}} .
$$

To be more specific, the canonical transformation is

$$
\begin{array}{ll}
q_{+}=\frac{1}{\sqrt{2}}\left(\sqrt{\frac{m \omega_{1}}{2}} x-\sqrt{\frac{2}{m \omega_{1}}} p_{y}\right), & p_{+}=\frac{1}{\sqrt{2}}\left(\sqrt{+\frac{m \omega_{1}}{2}} y+\sqrt{\frac{2}{m \omega_{1}}} p_{x}\right), \\
q_{-}=\frac{1}{\sqrt{2}}\left(\sqrt{\frac{m \omega_{1}}{2}} x+\sqrt{\frac{2}{m \omega_{1}}} p_{y}\right), & p_{-}=\frac{1}{\sqrt{2}}\left(-\sqrt{\frac{m \omega_{1}}{2}} y+\sqrt{\frac{2}{m \omega_{1}}} p_{x}\right), \\
q_{3}=\sqrt{m \omega_{z}} z, \quad p_{3}=\frac{1}{\sqrt{\omega_{m z}}} p_{z}, &
\end{array}
$$

and the Hamiltonian becomes

$$
H=\omega_{+} \cdot \frac{1}{2}\left(q_{+}^{2}+p_{+}^{2}\right)-\omega_{-} \cdot \frac{1}{2}\left(q_{-}^{2}+p_{-}^{2}\right)+\omega_{z} \cdot \frac{1}{2}\left(q_{3}^{2}+p_{3}^{2}\right) .
$$

Combining Eq. (2) with Eqs. (4)-(6) we obtain the new canonical coordinates and momenta in terms of the cartesian coordinates and velocities

$$
\begin{aligned}
& q_{+}=-\sqrt{\frac{m}{\omega_{1}}}\left(\dot{y}+\omega_{-} x\right), \quad p_{+}=\sqrt{\frac{m}{\omega_{1}}}\left(\dot{x}-\omega_{-} y\right), \\
& q_{-}=+\sqrt{\frac{m}{\omega_{1}}}\left(\dot{y}+\omega_{+} x\right), \quad p_{-}=\sqrt{\frac{m}{\omega_{1}}}\left(\dot{x}-\omega_{+} y\right), \\
& q_{3}=\sqrt{m \omega_{z}} z, \quad p_{3}=\sqrt{\frac{m}{\omega_{z}}} \dot{z} .
\end{aligned}
$$

When we compare these expressions to the "velocity vectors" $\vec{V}^{ \pm}$, which were introduced by Brown and Gabrielse [9] and which are frequently used in the literature,

$$
\vec{V}^{ \pm}=\mathbf{e}_{x} V_{x}^{ \pm}+\mathbf{e}_{y} V_{y}^{ \pm}=\dot{\vec{\rho}}+\omega_{\mp} \mathbf{e}_{z} \times \vec{\rho},
$$

with $\vec{\rho}=x \mathbf{e}_{x}+y \mathbf{e}_{y}$, it becomes evident that our canonical coordinates and momenta are equivalent to the components of these vectors. ${ }^{2}$ The relations are $V_{x}^{ \pm}=\sqrt{\omega_{1} / m} p_{ \pm}$and $V_{y}^{ \pm}=\mp \sqrt{\omega_{1} / m} q_{ \pm}$. Unfortunately, the notion of velocity vectors and the corresponding notation obscure the true dynamical meaning of these variables. For clarity it is therefore preferable to use the language of the canonical Hamiltonian theory.

The canonical formalism makes it easy to pass from the classical to a quantized description of the motion. One simply has to replace the classical Poisson brackets of the canonical variables $q_{i}$ and $p_{i}$, where $i=+,-, 3$, by commutator brackets for the corresponding

\footnotetext{
${ }^{2}$ The canonical properties of $V_{x}^{ \pm}, V_{y}^{ \pm}$were briefly mentioned by Brown and Gabrielse [9] and used by them for quantization. Most of the literature, however, ignores this important aspect.
} 
operators, $\left\{q_{i}, p_{j}\right\}=\delta_{i, j} \rightarrow\left[q_{i}, p_{j}\right]=i \hbar \delta_{i, j}$. As Eq. (7) shows the Hamiltonian of the ideal Penning trap is a composite of three harmonic oscillators, thus it is advantageous to introduce the familiar annihilation and creation operators for the oscillator quanta,

$$
a_{k}=\frac{1}{\sqrt{2 \hbar}}\left(q_{k}+i p_{k}\right), \quad a_{k}^{\dagger}=\frac{1}{\sqrt{2 \hbar}}\left(q_{k}-i p_{k}\right), \quad k=+,-, 3,
$$

obeying the commutation rules

$$
\left[a_{ \pm}, a_{ \pm}^{\dagger}\right]=1, \quad\left[a_{ \pm}, a_{\mp}^{\dagger}\right]=0, \quad\left[a_{ \pm}, a_{\mp}\right]=0 .
$$

In terms of these operators the Hamiltonian operator of the ideal Penning trap (7) becomes

$$
H=\hbar \omega_{+}\left(a_{+}^{\dagger} a_{+}+\frac{1}{2}\right)-\hbar \omega_{-}\left(a_{-}^{\dagger} a_{-}+\frac{1}{2}\right)+\hbar \omega_{z}\left(a_{3}^{\dagger} a_{3}+\frac{1}{2}\right),
$$

and the Heisenberg equations of motion $i \hbar\left(\mathrm{d} a_{i} / \mathrm{d} t\right)=\left[a_{i}, H\right]$ are easy to solve,

$$
a_{+}(t)=\mathrm{e}^{-\mathrm{i} \omega_{+} t} a_{+}(0), \quad a_{-}(t)=\mathrm{e}^{+\mathrm{i} \omega_{-} t} a_{-}(0), \quad a_{3}(t)=\mathrm{e}^{-\mathrm{i} \omega_{z} t} a_{3}(0) .
$$

The cartesian position and velocity operators are expressed in terms of the modal annihilation and creation operators by

$$
\begin{aligned}
& x=\frac{1}{\sqrt{m \omega_{1}}}\left(q_{+}+q_{-}\right)=\sqrt{\frac{\hbar}{2 m \omega_{1}}}\left(a_{+}+a_{+}^{\dagger}+a_{-}+a_{-}^{\dagger}\right), \\
& y=\frac{1}{\sqrt{m \omega_{1}}}\left(p_{+}-p_{-}\right)=-i \sqrt{\frac{\hbar}{2 m \omega_{1}}}\left(a_{+}-a_{+}^{\dagger}-a_{-}+a_{-}^{\dagger}\right), \\
& z=\frac{1}{\sqrt{m \omega_{z}}} q_{3}=\sqrt{\frac{\hbar}{2 m \omega_{z}}}\left(a_{3}+a_{3}^{\dagger}\right), \\
& \dot{x}=\frac{1}{\sqrt{m \omega_{1}}}\left(\omega_{+} p_{+}-\omega_{-} p_{-}\right)=-i \sqrt{\frac{\hbar}{2 m \omega_{1}}}\left(\omega_{+}\left(a_{+}-a_{+}^{\dagger}\right)-\omega_{-}\left(a_{-}-a_{-}^{\dagger}\right)\right), \\
& \dot{y}=-\frac{1}{\sqrt{m \omega_{1}}}\left(\omega_{+} q_{+}+\omega_{-} q_{-}\right)=-\sqrt{\frac{\hbar}{2 m \omega_{1}}}\left(\omega_{+}\left(a_{+}+a_{+}^{\dagger}\right)+\omega_{-}\left(a_{-}+a_{-}^{\dagger}\right)\right), \\
& \dot{z}=\frac{1}{\sqrt{m \omega_{z}}} p_{3}=-i \sqrt{\frac{\hbar}{2 m \omega_{z}}}\left(a_{3}-a_{3}^{\dagger}\right) .
\end{aligned}
$$

These relations are needed in order to calculate the ion trajectories inside the Penning trap.

\subsection{Interaction with the external quadrupole field}

The electric field present in the ideal Penning trap is derived from the scalar electric potential $\Phi_{0}(x, y, z)$ defined in Eq. (1). Additional electric fields may be introduced into the trap interior by dividing the ring electrode into several segments (usually 2,4 , or 8) and applying specific additional voltages to each of these segments and eventually also to the end electrodes. These voltages may be static or time dependent, most often they are periodic like $\cos \omega_{\mathrm{d}} t$, with the driving frequency $\nu_{\mathrm{d}}=\omega_{\mathrm{d}} / 2 \pi$ chosen to suit the intended purpose. For experimental details we refer to the literature $[10,15,16]$. For the theoretical treatment it is important that the sources of these additional fields are on the electrode surfaces, while the trap interior is free of sources. The additional fields can then be described in terms of potentials $\Phi_{1}(x, y, z, t)$ that are solutions of the boundary value problem defined by the electrodes and that satisfy a Laplace differential equation in the trap interior. The potentials therefore possess a series expansion in terms of harmonic polynomials (i.e., solutions of the Laplace equation), and near the trap center the first few terms suffice for an accurate description of the ion motion.

To simplify the discussion we assume that our electric field configuration is symmetric under reflections by the $x y$-plane, the $x z$-plane, and the $y z$-plane, and hence also under space reflections with respect to the origin (trap center) and under rotations by $180^{\circ}$ around the $z$-axis. Under these conditions the polynomial expansion of the electric potential $\Phi_{1}(x, y, z, t)$ involves only polynomials of even degree. This paper focuses on quadrupole fields deriving from the quadratic terms and disregards the anharmonic corrections due to 4th and higher degree terms. The most general polynomial solution of second degree of the Laplace equation compatibel with the above symmetry conditions is then given by

$$
\Phi_{1}(x, y, z, t)=C_{0}(t) \cdot\left(2 z^{2}-x^{2}-y^{2}\right)+C_{1}(t) \cdot\left(x^{2}-y^{2}\right)+\cdots .
$$


The first term would be present if we would apply an alternating voltage between the ring electrode and the two end electrodes, we would then obtain a combined Penning and Paul trap. This case is of no interest here, so we choose $C_{0}(t)=0$. The second term becomes relevant when we work with a ring electrode divided into four or eight segments and the electric potentials on the individual segments are chosen such that (i) opposite segments are on the same potential and (ii) $\sum c_{i} V_{i}=0$, where $c_{i}$ denotes the angle over which the $i$ th segment extends, $V_{i}$ is the potential applied to the $i$ th segment, and the sum extends over all four or eight segments, respectively. The exact value of the coefficient $C_{1}$ depends on details of the trap geometry, in particular on the values $c_{i}$ and on the strength of the higher order anharmonic terms. Summarizing this discussion we shall work from here on with the potential

$$
\Phi_{1}(x, y, z, t)=C_{1} \cos \left(\phi_{\mathrm{d}}(t)\right) \cdot\left(x^{2}-y^{2}\right),
$$

where $\phi_{\mathrm{d}}(t)=\omega_{\mathrm{d}} t+\chi_{\mathrm{d}}$ describes the phase of the external quadrupole field. In polar coordinates $x=r \cos \varphi, y=r \sin \varphi$ this potential becomes

$$
\Phi_{1}(x, y, z, t)=C_{1} \cos \left(\phi_{\mathrm{d}}(t)\right) \cdot r^{2} \cos 2 \varphi=\frac{1}{2} C_{1} \cdot r^{2} \cdot\left[\cos \left(2 \varphi+\omega_{\mathrm{d}} t+\chi_{\mathrm{d}}\right)+\cos \left(2 \varphi-\omega_{\mathrm{d}} t-\chi_{\mathrm{d}}\right)\right] .
$$

Here the first term represents a potential rotating in the clockwise sense, while the second term is a potential rotating anti-clockwise.

To study the ion motion under the influence of this additional alternating quadrupole field we need to add $q \Phi_{1}(x, y, z, t)$ to the Hamiltonian of the ideal Penning trap and to solve the resultant equations of motion. The term $q \Phi_{1}$ then denotes the additional potential energy the ion has when its instantaneous position at time $t$ is at $x, y, z$. Therefore the arguments of $q \Phi_{1}(x, y, z, t)$ do no longer refer to a general space-time point, but to the space position of the ion at time $t$. The azimuthal motion of an ion in an ideal Penning trap is described by a superposition of clockwise rotations, $x(t)+i y(t)=r(t) \mathrm{e}^{\mathrm{i} \varphi(t)}=A_{+} \mathrm{e}^{-\mathrm{i} \omega_{+} t}+A_{-} \mathrm{e}^{-\mathrm{i} \omega_{-} t}$, where $A_{+}$and $A_{-}$ are complex amplitudes. After insertion into Eq. (24) of $r^{2} \cos 2 \varphi=\Re\left[(x+i y)^{2}\right]=\Re\left[A_{+}^{2} \mathrm{e}^{-2 i \omega_{+} t}+A_{-}^{2} \mathrm{e}^{-2 i \omega_{-} t}+2 A_{+} A_{-} \mathrm{e}^{-\mathrm{i} \omega_{\mathrm{c}} t}\right]$, using $\omega_{+}+\omega_{-}=\omega_{\mathrm{c}}$, we observe that the second term in (24) contains only very rapidly oscillating contributions that approximately average out to zero. Therefore, in the spirit of the "rotating wave approximation" [17] we drop the second term from further consideration. Obviously there are three ranges of driving frequencies for the additional quadrupole field where the potential (24) could become a slowly varying function of time and thus lead to important effects, namely $\omega_{\mathrm{d}} \approx 2 \omega_{+}, \omega_{\mathrm{d}} \approx 2 \omega_{-}$, and $\omega_{\mathrm{d}} \approx \omega_{\mathrm{c}}$. By choosing the driving frequency near one of these three values we can determine which transition is driven by the rf-quadrupole field.

The physics behind this becomes much more clear when we consider the problem in the quantum mechanical framework. The coordinates $x, y, z$ must then be considered not as real numbers, but as the Hilbert space operators associated with the position of the ion. Just like the classical coordinates these operators can be transformed into general modal operators $q_{+}, q_{-}, q_{3}$ and $p_{+}, p_{-}$, $p_{3}$ according to Eqs. (4)-(6). Next we express everything in terms of modal creation and annihilation operators using Eq. (12). One finds for the additional potential energy in the rotating wave approximation

$$
q \Phi_{1}(x, y, z, t)=\frac{q \hbar C_{1}}{2 m \omega_{1}}\left\{+\exp \left[-\mathrm{i} \phi_{\mathrm{d}}(t)\right] \cdot\left(a_{+}^{\dagger 2}+a_{-}^{2}+2 a_{+}^{\dagger} a_{-}\right)+\exp \left[\mathrm{i} \phi_{\mathrm{d}}(t)\right] \cdot\left(a_{+}^{2}+a_{-}^{\dagger 2}+2 a_{-}^{\dagger} a_{+}\right)\right\} .
$$

The first term describes transitions in which one photon of energy $\hbar \omega_{\mathrm{d}} \approx 2 \hbar \omega_{+}$is absorbed from the driving quadrupole field and two excitation quanta of the modified cyclotron oscillator are created, the next term describes transitions in which one photon of energy $\hbar \omega_{\mathrm{d}} \approx 2 \hbar \omega_{-}$is absorbed from the driving quadrupole field and two excitation quanta of the magnetron oscillator are annihilated, finally the third term describes the conversion of a magnetron quantum into a modified cyclotron quantum, with simultaneous absorption of a photon of energy $\hbar \omega_{\mathrm{c}}$. The last three terms describe the respective inverse transitions. Only the interconversion of modes is of interest for our further investigation, therefore the interaction of the ion with the rf-quadrupole field is reduced to

$$
H_{1}(t)=\hbar g\left(\mathrm{e}^{-\mathrm{i} \phi_{\mathrm{d}}(t)} a_{+}^{\dagger}(t) a_{-}(t)+\mathrm{e}^{+\mathrm{i} \phi_{\mathrm{d}}(t)} a_{-}^{\dagger}(t) a_{+}(t)\right)
$$

with a driving frequency $\omega_{\mathrm{d}} \approx \omega_{\mathrm{c}}$ and the coupling constant $g=q C_{1} /\left(2 m \omega_{1}\right)$.

\subsection{Ion motion in the presence of the external quadrupole field}

For the remainder of this paper we shall disregard the axial coordinate $z$ and the axial oscillator mode, because the axial mode is not coupled to the magnetron and modified cyclotron modes by the external quadrupole field applied to the ring segments, see Eq. (26). Thus it is sufficient to study the simplified Hamiltonian

$$
H(t)=\hbar \omega_{+}\left(a_{+}^{\dagger} a_{+}+\frac{1}{2}\right)-\hbar \omega_{-}\left(a_{-}^{\dagger} a_{-}+\frac{1}{2}\right)+\hbar g\left(\mathrm{e}^{-\mathrm{i} \phi_{\mathrm{d}}(t)} a_{+}^{\dagger}(t) a_{-}(t)+\mathrm{e}^{+\mathrm{i} \phi_{\mathrm{d}}(t)} a_{-}^{\dagger}(t) a_{+}(t)\right) .
$$

We shall make use of the Heisenberg picture, where the specific physical system is described by a time independent state vector, or more generally a time independent density matrix, and where its dynamical development in time is reflected in the time dependence of the observables and other Hilbert space operators, as for example in the present problem the creation and annihilation operators of the modified cyclotron and magnetron modes. Our analysis starts with the statement of Heisenberg's equations of motion for the 
annihilation operators $a_{+}(t)$ and $a_{-}(t)$ of the quanta of the modified cyclotron oscillator and the magnetron oscillator,

$$
\begin{aligned}
& i \hbar \frac{\mathrm{d}}{\mathrm{d} t} a_{+}(t)=\left[a_{+}(t), H(t)\right]=+\hbar \omega_{+} a_{+}(t)+\hbar g \mathrm{e}^{-\mathrm{i} \phi_{\mathrm{d}}(t)} a_{-}(t), \\
& i \hbar \frac{\mathrm{d}}{\mathrm{d} t} a_{-}(t)=\left[a_{-}(t), H(t)\right]=-\hbar \omega_{-} a_{-}(t)+\hbar g \mathrm{e}^{+\mathrm{i} \phi_{\mathrm{d}}(t)} a_{+}(t) .
\end{aligned}
$$

Equations for the creation operators are obtained by taking the adjoints of these equations. The Eqs. (28) and (29) are coupled linear differential equations with time dependent coefficients, due to the time dependence of the external quadrupole field. Fortunately it is possible to eliminate the time dependence of the coefficients by a unitary transformation in Hilbert space,

$$
U(t)=\exp \left[-\frac{i}{2} \omega_{1} t \cdot\left(a_{+}^{\dagger}(t) a_{+}(t)+a_{-}^{\dagger}(t) a_{-}(t)\right)-\frac{i}{2} \phi_{\mathrm{d}}(t) \cdot\left(a_{+}^{\dagger}(t) a_{+}(t)-a_{-}^{\dagger}(t) a_{-}(t)\right)\right] .
$$

We define new operators

$$
\begin{aligned}
& a_{+}^{\prime}(t)=U(t) a_{+}(t) U^{\dagger}(t)=\exp \left(+\frac{i}{2}\left(\omega_{1} t+\phi_{\mathrm{d}}(t)\right)\right) \cdot a_{+}(t), \\
& a_{-}^{\prime}(t)=U(t) a_{-}(t) U^{\dagger}(t)=\exp \left(+\frac{i}{2}\left(\omega_{1} t-\phi_{\mathrm{d}}(t)\right)\right) \cdot a_{-}(t),
\end{aligned}
$$

where in the second part of the equations we have used the Baker-Campbell-Hausdorff identities to evaluate the action of the unitary transformation on the annihilation operators $a_{+}(t)$ and $a_{-}(t)$. From Heisenberg's equations of motion (28) and (29) we obtain differential equations for $a_{+}^{\prime}(t)$ and $a_{-}^{\prime}(t)$,

$$
\begin{aligned}
\frac{\mathrm{d}}{\mathrm{d} t} a_{+}^{\prime}(t) & =-\frac{i}{2}\left(-\delta \cdot a_{+}^{\prime}(t)+2 g \cdot a_{-}^{\prime}(t)\right), \\
\frac{\mathrm{d}}{\mathrm{d} t} a_{-}^{\prime}(t) & =-\frac{i}{2}\left(+2 g \cdot a_{+}^{\prime}(t)+\delta \cdot a_{-}^{\prime}(t)\right) .
\end{aligned}
$$

As desired these equations are linear differential equations with constant coefficients. The eigenvalues of the system are pure imaginary, $\lambda_{1,2}= \pm(i / 2) \sqrt{\delta^{2}+4 g^{2}}= \pm(i / 2) \omega_{\mathrm{R}}$, consequently the solutions are periodic functions. The quantity $\omega_{\mathrm{R}}=\sqrt{\delta^{2}+4 g^{2}}$ is defined by us in analogy to nuclear-magnetic-resonance as the (circular) Rabi frequency of the system, it quantifies the frequency of the periodic conversion of the magnetron mode into the modified cyclotron mode and vice versa, as shall be seen later on. The system of differential Eqs. (33) and (34) can be solved by the standard method of exponentiation. For this purpose it is convenient to combine the two annihilation operators into a two-component spinor-like object, so that their coupled equations can be formulated and solved in terms of complex $2 \times 2$ matrices, making use of the Pauli matrices $\sigma_{1}, \sigma_{2}, \sigma_{3}$. Thus the unitary transformation of Eqs. (31) and (32) is written as

$$
\left(\begin{array}{l}
a_{+}^{\prime}(t) \\
a_{-}^{\prime}(t)
\end{array}\right)=\mathrm{e}^{(i / 2) \omega_{1} t} \mathcal{N}\left(\phi_{\mathrm{d}}(t)\right)\left(\begin{array}{l}
a_{+}(t) \\
a_{-}(t)
\end{array}\right)
$$

with

$$
\mathcal{N}(t)=\left(\begin{array}{cc}
\mathrm{e}^{(i / 2) \phi_{\mathrm{d}}(t)} & 0 \\
0 & \mathrm{e}^{-(i / 2) \phi_{\mathrm{d}}(t)}
\end{array}\right)=\exp \left[\frac{i}{2} \sigma_{3} \cdot \phi_{\mathrm{d}}(t)\right] .
$$

For later use we also note that

$$
\mathcal{N}\left(t_{0}+\tau\right)=\mathrm{e}^{(i / 2) \sigma_{3} \omega_{\mathrm{d}} \tau} \mathcal{N}\left(\phi_{\mathrm{d}}\left(t_{0}\right)\right)=\left(\begin{array}{cc}
\mathrm{e}^{(i / 2) \omega_{\mathrm{d}} \tau} & 0 \\
0 & \mathrm{e}^{-(i / 2) \omega_{\mathrm{d}} \tau}
\end{array}\right) \mathcal{N}\left(\phi_{\mathrm{d}}\left(t_{0}\right)\right) .
$$

The transformed Heisenberg Eqs. (33) and (34) read in matrix notation

$$
\frac{\mathrm{d}}{\mathrm{d} t}\left(\begin{array}{c}
a_{+}^{\prime}(t) \\
a_{-}^{\prime}(t)
\end{array}\right)=-\frac{i}{2}\left(\begin{array}{cc}
-\delta & 2 g \\
2 g & +\delta
\end{array}\right)\left(\begin{array}{c}
a_{+}^{\prime}(t) \\
a_{-}^{\prime}(t)
\end{array}\right)=-\frac{i}{2}\left(2 g \cdot \sigma_{1}-\delta \cdot \sigma_{3}\right)\left(\begin{array}{c}
a_{+}^{\prime}(t) \\
a_{-}^{\prime}(t)
\end{array}\right) .
$$


They can be solved immediately by exponentiation and the time development of the system during the time interval $t_{0} \leq t \leq t_{0}+\tau$ is described by a complex $2 \times 2$ matrix $\mathcal{M}(\tau ; \delta, g)$

$$
\left(\begin{array}{l}
a_{+}^{\prime}\left(t_{0}+\tau\right) \\
a_{-}^{\prime}\left(t_{0}+\tau\right)
\end{array}\right)=\mathcal{M}(\tau ; \delta, g)\left(\begin{array}{c}
a_{+}^{\prime}\left(t_{0}\right) \\
a_{-}^{\prime}\left(t_{0}\right)
\end{array}\right) .
$$

The anticommutation properties of the Pauli matrices yield the relation $\left(2 g \cdot \sigma_{1}-\delta \cdot \sigma_{3}\right)^{2}=4 g^{2}+\delta^{2}=\omega_{\mathrm{R}}^{2}$, thus the matrix $\mathcal{M}$ is calculated as

$$
\mathcal{M}(\tau ; \delta, g)=\exp \left[-\frac{i}{2}\left(2 g \cdot \sigma_{1}-\delta \cdot \sigma_{3}\right) \tau\right]=\cos \left(\frac{\omega_{\mathrm{R}} \tau}{2}\right)-i \sin \left(\frac{\omega_{\mathrm{R}} \tau}{2}\right)\left(\frac{2 g}{\omega_{\mathrm{R}}} \cdot \sigma_{1}-\frac{\delta}{\omega_{\mathrm{R}}} \cdot \sigma_{3}\right),
$$

or in explicite matrix form

$$
\mathcal{M}(\tau ; \delta, g)=\left(\begin{array}{cc}
\cos \left(\frac{\omega_{\mathrm{R}} \tau}{2}\right)+i \frac{\delta}{\omega_{\mathrm{R}}} \sin \left(\frac{\omega_{\mathrm{R}} \tau}{2}\right) & -i \frac{2 g}{\omega_{\mathrm{R}}} \sin \left(\frac{\omega_{\mathrm{R}} \tau}{2}\right) \\
-i \frac{2 g}{\omega_{\mathrm{R}}} \sin \left(\frac{\omega_{\mathrm{R}} \tau}{2}\right) & \cos \left(\frac{\omega_{\mathrm{R}} \tau}{2}\right)-i \frac{\delta}{\omega_{\mathrm{R}}} \sin \left(\frac{\omega_{\mathrm{R}} \tau}{2}\right)
\end{array}\right) .
$$

Collecting all results we can state our final result for the time development of the creation and annihilation operators during the time interval $t_{0} \leq t \leq t_{0}+\tau$ as

$$
\left(\begin{array}{l}
a_{+}\left(t_{0}+\tau\right) \\
a_{-}\left(t_{0}+\tau\right)
\end{array}\right)=\mathrm{e}^{-(i / 2) \omega_{1} \tau} \mathcal{N}^{-1}\left(\phi_{\mathrm{d}}\left(t_{0}+\tau\right)\right) \mathcal{M}(\tau ; \delta, g) \mathcal{N}\left(\phi_{\mathrm{d}}\left(t_{0}\right)\right)\left(\begin{array}{l}
a_{+}\left(t_{0}\right) \\
a_{-}\left(t_{0}\right)
\end{array}\right) .
$$

\subsection{Classical ion trajectories}

Shortly after the discovery of wave mechanics Schrödinger found Gaussian wave packet solutions of the harmonic oscillator wave equation that stayed together without spreading and followed classical trajectories [18]. These states of minimal uncertainty represent the closest possible approximation to classical mechanics, they have become known under the name "coherent states" [17]. They can be expanded in terms of Fock states (number states). For any given complex number $\alpha$ we have

$$
|\alpha\rangle=\mathrm{e}^{-|\alpha|^{2} / 2} \sum_{n=0}^{\infty} \frac{\alpha^{n}}{\sqrt{n !}}|n\rangle=\mathrm{e}^{-|\alpha|^{2} / 2} \sum_{n=0}^{\infty} \frac{\left(\alpha a^{\dagger}\right)^{n}}{n !}|0\rangle=\mathrm{e}^{-|\alpha|^{2} / 2} \exp \left[\alpha a^{\dagger}\right]|0\rangle,
$$

where $|0\rangle$ denotes the ground state, $|n\rangle$ is the Fock state representing $n$ oscillator quanta, and $\mathrm{e}^{-|\alpha|^{2} / 2}$ is the normalization factor. The coherent state $|\alpha\rangle$ can be uniquely characterized as the normalized eigenstate of the harmonic oscillator annihilation operator $a$ with a complex eigenvalue $\alpha$,

$$
a|\alpha\rangle=\alpha|\alpha\rangle .
$$

For the classical description of the ion motion in a Penning trap one defines the coherent states

$$
\left|\alpha_{+}(0), \alpha_{-}(0)\right\rangle=\mathrm{e}^{-\left(\left|\alpha_{+}(0)\right|^{2}+\left|\alpha_{-}(0)\right|^{2}\right) / 2} \sum_{m=0}^{\infty} \sum_{n=0}^{\infty} \frac{\alpha_{+}^{m}(0) \alpha_{-}^{n}(0)}{\sqrt{m ! n !}}|m, n\rangle,
$$

where $\alpha_{+}(0)=\left|\alpha_{+}(0)\right| \mathrm{e}^{-\mathrm{i} \chi_{+}}$and $\alpha_{-}(0)=\left|\alpha_{-}(0)\right| \mathrm{e}^{+\mathrm{i} \chi_{-}}$are given complex numbers [8]. They may be considered as initial values that are related to the cartesian initial values of a classical ion trajectory by Eqs. (16)-(21). With given initial values we calculate the expectation values of the annihilation operators $a_{+}(t)$ and $a_{-}(t)$, which are the solutions of the Heisenberg equations of motion (28) and (29) and are explicitely given in Eq. (42). In this way we obtain two complex functions of time

$$
\alpha_{ \pm}(t)=\left\langle\alpha_{+}(0) \alpha_{-}(0)\left|a_{ \pm}(t)\right| \alpha_{+}(0) \alpha_{-}(0)\right\rangle,
$$

which represent the complex oscillator amplitudes of the modified cyclotron and magnetron oscillators at time $t$. Working out the details we obtain

$$
\begin{aligned}
& \alpha_{+}(t)=\mathrm{e}^{-\mathrm{i}\left(\omega_{+}+\delta / 2\right) t}\left[\left(\cos \frac{\omega_{\mathrm{R}} t}{2}+i \frac{\delta}{\omega_{\mathrm{R}}} \sin \frac{\omega_{\mathrm{R}} t}{2}\right) \alpha_{+}(0)-i \frac{2 g}{\omega_{\mathrm{R}}} \sin \frac{\omega_{\mathrm{R}} t}{2} \mathrm{e}^{-\mathrm{i} \chi_{\mathrm{d}}} \alpha_{-}(0)\right], \\
& \alpha_{-}(t)=\mathrm{e}^{+\mathrm{i}\left(\omega_{-}+\delta / 2\right) t}\left[-i \frac{2 g}{\omega_{\mathrm{R}}} \sin \frac{\omega_{\mathrm{R}} t}{2} \mathrm{e}^{+\mathrm{i} \chi_{\mathrm{d}}} \alpha_{+}(0)+\left(\cos \frac{\omega_{\mathrm{R}} t}{2}-i \frac{\delta}{\omega_{\mathrm{R}}} \sin \frac{\omega_{\mathrm{R}} t}{2}\right) \alpha_{-}(0)\right] .
\end{aligned}
$$


For the practical application of these results it is useful to know that the instantaneous radii for the modified cyclotron and the magnetron motion are given by

$$
R_{+}(t)=\sqrt{\frac{2 \hbar}{m \omega_{1}}}\left|\alpha_{+}(t)\right| \quad R_{-}(t)=\sqrt{\frac{2 \hbar}{m \omega_{1}}}\left|\alpha_{-}(t)\right| .
$$

Thus, with $\Delta=\chi_{+}+\chi_{-}-\chi_{\mathrm{d}}$ the time-dependence of the radii is given by

$$
\begin{aligned}
R_{ \pm}^{2}(t)= & \left(\cos ^{2} \frac{\omega_{\mathrm{R}} t}{2}+\frac{\delta^{2}}{\omega_{\mathrm{R}}^{2}} \sin ^{2} \frac{\omega_{\mathrm{R}} t}{2}\right) R_{ \pm}^{2}(0)+\frac{4 g^{2}}{\omega_{\mathrm{R}}^{2}} \sin ^{2} \frac{\omega_{\mathrm{R}} t}{2} R_{\mp}^{2}(0) \pm 2 \cdot \frac{2 g}{\omega_{\mathrm{R}}} \sin \frac{\omega_{\mathrm{R}} t}{2} \cdot\left(\cos \frac{\omega_{\mathrm{R}} t}{2} \sin \Delta-\frac{\delta}{\omega_{\mathrm{R}}} \sin \frac{\omega_{\mathrm{R}} t}{2} \cos \Delta\right) \\
& \times R_{+}(0) R_{-}(0),
\end{aligned}
$$

which is equivalent to the result found by König et al. [20].

The transformation Eqs. (16) and (17) then tell us how to relate the complex oscillator amplitudes to the cartesian coordinates,

$$
\langle x(t)+i y(t)\rangle=\left\langle\alpha_{+}(0) \alpha_{-}(0)|x(t)+i y(t)| \alpha_{+}(0) \alpha_{-}(0)\right\rangle=\sqrt{\frac{2 \hbar}{m \omega_{1}}}\left(\alpha_{+}(t)+\alpha_{-}^{*}(t)\right),
$$

where the asterisk denotes complex conjugation. In real form the parametric representation of the ion trajectory is

$$
\begin{aligned}
\langle x(t)\rangle & =\sqrt{\frac{2 \hbar}{m \omega_{1}}}\left(\Re\left(\alpha_{+}(t)\right)+\Re\left(\alpha_{-}(t)\right)\right), \\
\langle y(t)\rangle & =\sqrt{\frac{2 \hbar}{m \omega_{1}}}\left(\Im\left(\alpha_{+}(t)\right)-\Im\left(\alpha_{-}(t)\right)\right) .
\end{aligned}
$$

Inserting our solution (42) we arrive at an explicite representation of the ion trajectories

$$
\begin{aligned}
\langle x(t)+i y(t)\rangle= & \mathrm{e}^{(-i / 2) \delta t} \sqrt{\frac{2 \hbar}{m \omega_{1}}} \cdot\left[\left(\cos \frac{\omega_{\mathrm{R}} t}{2}+i \frac{\delta}{\omega_{\mathrm{R}}} \sin \frac{\omega_{\mathrm{R}} t}{2}\right) \cdot\left(\mathrm{e}^{-\mathrm{i} \omega_{+} t} \alpha_{+}(0)+\mathrm{e}^{-\mathrm{i} \omega_{-} t} \alpha_{-}^{*}(0)\right)\right. \\
& \left.-i \mathrm{e}^{-\mathrm{i} \chi_{\mathrm{d}}} \cdot \frac{2 g}{\omega_{\mathrm{R}}} \sin \frac{\omega_{\mathrm{R}} t}{2}\left(\mathrm{e}^{-\mathrm{i} \omega_{+} t} \alpha_{-}(0)-\mathrm{e}^{-\mathrm{i} \omega_{-} t} \alpha_{+}^{*}(0)\right)\right]
\end{aligned}
$$

For a graphical representation of ion trajectories calculated by this approach see Fig. 9 below. These figures correspond to those obtained by Bollen et al. by numerical integration of the equations of motion [15].

\subsection{The Bloch vector operator}

\subsubsection{Basic definition and properties}

Following [8] we now introduce the Bloch vector operator $\mathbf{T}$, which is a vector in some abstract three-dimensional space with components that are linear operators acting on the infinite dimensional Hilbert space of our system:

$$
\mathbf{T}=\mathbf{e}_{1} \cdot T_{1}+\mathbf{e}_{2} \cdot T_{2}+\mathbf{e}_{3} \cdot T_{3}
$$

with

$$
T_{1}=\frac{1}{2}\left(a_{+}^{\dagger} a_{-}+a_{-}^{\dagger} a_{+}\right), \quad T_{2}=\frac{1}{2 i}\left(a_{+}^{\dagger} a_{-}-a_{-}^{\dagger} a_{+}\right), \quad T_{3}=\frac{1}{2}\left(a_{+}^{\dagger} a_{+}-a_{-}^{\dagger} a_{-}\right) .
$$

An expectation value of the Bloch vector operator $\langle\mathbf{T}\rangle=\operatorname{Tr}\{\rho \mathbf{T}\}$, calculated with some density matrix $\rho$, is an ordinary three-vector with real components and shall simply be referred to as "Bloch vector". Using the equal time commutation rules (13) it is easy to verify that

$$
\left[T_{1}, T_{2}\right]=i T_{3}, \quad\left[T_{3}, T_{1}\right]=i T_{2}, \quad\left[T_{2}, T_{3}\right]=i T_{1} .
$$

The components of the Bloch vector operator therefore satisfy commutation rules analogous to those of an angular momentum operator, in mathematical language, they span the Lie algebra of the group $S U(2)$. This set of operators is completed by

$$
T_{0}=\frac{1}{2}\left(a_{+}^{\dagger} a_{+}+a_{-}^{\dagger} a_{-}\right),
$$


which commutes with $T_{1}, T_{2}, T_{3}$, that is $\left[T_{0}, T_{i}\right]=0$ for $i=1,2,3$. Denoting the operators for the total number of quanta in the modified cyclotron and magnetron oscillators by $N_{+}=a_{+}^{\dagger} a_{+}$and $N_{-}=a_{-}^{\dagger} a_{-}$respectively, we see that

$$
N_{+}=T_{0}+T_{3}, \quad N_{-}=T_{0}-T_{3}, \quad N_{\text {tot }}=N_{+}+N_{-}=2 T_{0},
$$

where $N_{\text {tot }}$ represents the total number of quanta in the system. It is also useful to note that

$$
\mathbf{T}^{2}=T_{1}^{2}+T_{2}^{2}+T_{3}^{2}=T_{0}\left(T_{0}+1\right) .
$$

The commutation relations imply $\left[\mathbf{T}^{2}, T_{i}\right]=0$ for $i=1,2,3$, and with the Eq. (63) it is easily shown that $\mathbf{T}^{2}$ represents a conserved quantity.

It is important that the Bloch vector operator may be used to reformulate the Hamiltonian operator Eq. (14),

$$
H(t)=\hbar \omega_{1}\left(T_{0}+\frac{1}{2}\right)+\hbar \omega_{\mathrm{c}} T_{3}(t)+\hbar \cdot 2 g\left(\cos \phi_{\mathrm{d}}(t) \cdot T_{1}(t)+\sin \phi_{\mathrm{d}}(t) \cdot T_{2}(t)\right) .
$$

Obviously $\left[T_{0}, H(t)\right]=0$, therefore the operator $T_{0}$ may be treated as constant, expressing the fact that the total number of quanta in the system is left unchanged by the quadrupole interaction. An equivalent conservation law formulated in different terms was first pointed out by Guan Shenheng and Marshall [19].

The concept of the Bloch vector is useful for us in several respects:

(a) The expectation value of $T_{3}$ is directly related to the expectation value for the energy in the cyclotron motional mode, which is the interesting quantity for Penning trap mass spectrometry,

$$
\left\langle E_{+}(t)\right\rangle=\hbar \omega_{+}\left\langle N_{+}(t)\right\rangle=\hbar \omega_{+}\left\langle a_{+}(t)^{\dagger} a_{+}(t)\right\rangle=\hbar \omega_{+}\left\langle T_{0}+T_{3}(t)\right\rangle .
$$

(b) The Bloch vector establishes a close analogy to the theoretical formalism of nuclear-magnetic-resonance (NMR) and thus to the origin of Ramsey's method of separated oscillating fields.

\subsubsection{Bloch vector operator: Equations of motion and their solution}

We now discuss the Heisenberg equations of motion for $\mathbf{T}$ that follow from the Hamiltonian Eq. (61). These are

$$
\begin{aligned}
\frac{\mathrm{d}}{\mathrm{d} t} T_{1}(t) & =(i \hbar)^{-1}\left[T_{1}(t), H(t)\right]=-\omega_{\mathrm{c}} T_{2}(t)+2 g \sin \phi_{\mathrm{d}}(t) T_{3}(t), \\
\frac{\mathrm{d}}{\mathrm{d} t} T_{2}(t) & =(i \hbar)^{-1}\left[T_{2}(t), H(t)\right]=+\omega_{\mathrm{c}} T_{1}(t)-2 g \cos \phi_{\mathrm{d}}(t) T_{3}(t), \\
\frac{\mathrm{d}}{\mathrm{d} t} T_{3}(t)=(i \hbar)^{-1}\left[T_{3}(t), H(t)\right] & =-2 g \sin \phi_{\mathrm{d}}(t) T_{1}(t)+2 g \cos \phi_{\mathrm{d}}(t) T_{2}(t) .
\end{aligned}
$$

These equations for the components of the Bloch vector are linear differential equations with time dependent coefficients. To obtain a sytem of differential equations with constant coefficients we tranform to a reference frame rotating counterclockwise about the three-axis with the angular velocity $\omega_{\mathrm{d}}$ :

$$
\mathbf{e}_{1}^{\prime}(t)=+\cos \phi_{\mathrm{d}}(t) \mathbf{e}_{1}+\sin \phi_{\mathrm{d}}(t) \mathbf{e}_{2}, \quad \mathbf{e}_{2}^{\prime}(t)=-\sin \phi_{\mathrm{d}}(t) \mathbf{e}_{1}+\cos \phi_{\mathrm{d}}(t) \mathbf{e}_{2}, \quad \mathbf{e}_{3}^{\prime}(t)=\mathbf{e}_{3} .
$$

The components $T_{i}^{\prime}$ of the Bloch vector in the rotating frame follow from

$$
\mathbf{T}(t)=\mathbf{e}_{1} T_{1}(t)+\mathbf{e}_{2} T_{2}(t)+\mathbf{e}_{3} T_{3}(t)=\mathbf{e}_{1}^{\prime}(t) T_{1}^{\prime}(t)+\mathbf{e}_{2}^{\prime}(t) T_{2}^{\prime}(t)+\mathbf{e}_{3}^{\prime}(t) T_{3}^{\prime}(t) .
$$

In matrix notation they are given by

$$
\left(\begin{array}{c}
T_{1}^{\prime}(t) \\
T_{2}^{\prime}(t) \\
T_{3}^{\prime}(t)
\end{array}\right)=\mathrm{N}\left(\phi_{\mathrm{d}}(t)\right)\left(\begin{array}{c}
T_{1}(t) \\
T_{2}(t) \\
T_{3}(t)
\end{array}\right)=\left(\begin{array}{ccc}
\cos \phi_{\mathrm{d}}(t) & \sin \phi_{\mathrm{d}}(t) & 0 \\
-\sin \phi_{\mathrm{d}}(t) & \cos \phi_{\mathrm{d}}(t) & 0 \\
0 & 0 & 1
\end{array}\right)\left(\begin{array}{c}
T_{1}(t) \\
T_{2}(t) \\
T_{3}(t)
\end{array}\right) .
$$

With the detuning $\delta=\omega_{\mathrm{d}}-\omega_{\mathrm{c}}$ we now derive from Eq. (63) the equations of motion in the rotating frame

$$
\frac{\mathrm{d}}{\mathrm{d} t}\left(\begin{array}{c}
T_{1}^{\prime}(t) \\
T_{2}^{\prime}(t) \\
T_{3}^{\prime}(t)
\end{array}\right)=\left(\begin{array}{ccc}
0 & \delta & 0 \\
-\delta & 0 & -2 g \\
0 & 2 g & 0
\end{array}\right)\left(\begin{array}{c}
T_{1}^{\prime}(t) \\
T_{2}^{\prime}(t) \\
T_{3}^{\prime}(t)
\end{array}\right) .
$$


We now have obtained linear differential equations with constant coefficients that can be solved by standard methods. The equations describe a precessional motion of the Bloch vector with an angular frequency $\omega_{\mathrm{R}}=\sqrt{\delta^{2}+4 g^{2}}$ (Rabi frequency) about the axis defined by the unit vector $\mathbf{e}_{1}^{\prime \prime}=\left(2 g / \omega_{\mathrm{R}}\right) \mathbf{e}_{1}^{\prime}-\left(\delta / \omega_{\mathrm{R}}\right) \mathbf{e}_{3}^{\prime}$, as can be determined from the factorization

$$
\left(\begin{array}{ccc}
0 & \delta & 0 \\
-\delta & 0 & -2 g \\
0 & 2 g & 0
\end{array}\right)=\left(\begin{array}{ccc}
\frac{2 g}{\omega_{\mathrm{R}}} & 0 & \frac{\delta}{\omega_{\mathrm{R}}} \\
0 & 1 & 0 \\
-\frac{\delta}{\omega_{\mathrm{R}}} & 0 & \frac{2 g}{\omega_{\mathrm{R}}}
\end{array}\right)\left(\begin{array}{ccc}
0 & 0 & 0 \\
0 & 0 & -\omega_{\mathrm{R}} \\
0 & \omega_{\mathrm{R}} & 0
\end{array}\right)\left(\begin{array}{ccc}
\frac{2 g}{\omega_{\mathrm{R}}} & 0 & -\frac{\delta}{\omega_{\mathrm{R}}} \\
0 & 1 & 0 \\
\frac{\delta}{\omega_{\mathrm{R}}} & 0 & \frac{2 g}{\omega_{\mathrm{R}}}
\end{array}\right) .
$$

The complete and exact solution of the equations of motion in the rotating frame (67) can now be written down in the form

$$
\left(\begin{array}{l}
T_{1}^{\prime}\left(t_{1}\right) \\
T_{2}^{\prime}\left(t_{1}\right) \\
T_{3}^{\prime}\left(t_{1}\right)
\end{array}\right)=\mathrm{M}\left(t_{1}-t_{0} ; \delta, g\right)\left(\begin{array}{l}
T_{1}^{\prime}\left(t_{0}\right) \\
T_{2}^{\prime}\left(t_{0}\right) \\
T_{3}^{\prime}\left(t_{0}\right)
\end{array}\right),
$$

where the matrix $\mathrm{M}$ describes time development of the system during the time interval $\tau=t_{1}-t_{0}$

$$
\mathrm{M}(\tau ; \delta, g)=\left(\begin{array}{ccc}
1+\frac{\delta^{2}}{\omega_{\mathrm{R}}^{2}}\left(\cos \omega_{\mathrm{R}} \tau-1\right) & +\frac{\delta}{\omega_{\mathrm{R}}} \sin \omega_{\mathrm{R}} \tau & \frac{2 g \delta}{\omega_{\mathrm{R}}^{2}}\left(\cos \omega_{\mathrm{R}} \tau-1\right) \\
-\frac{\delta}{\omega_{\mathrm{R}}} \sin \omega_{\mathrm{R}} \tau & \cos \omega_{\mathrm{R}} \tau & -\frac{2 g}{\omega_{\mathrm{R}}} \sin \omega_{\mathrm{R}} \tau \\
\frac{2 g \delta}{\omega_{\mathrm{R}}^{2}}\left(\cos \omega_{\mathrm{R}} \tau-1\right) & +\frac{2 g}{\omega_{\mathrm{R}}} \sin \omega_{\mathrm{R}} \tau & 1+\frac{4 g^{2}}{\omega_{\mathrm{R}}^{2}}\left(\cos \omega_{\mathrm{R}} \tau-1\right)
\end{array}\right) .
$$

Note that we have $\mathrm{M}\left(\tau_{1} ; \delta, g\right) \cdot \mathrm{M}\left(\tau_{2} ; \delta, g\right)=\mathrm{M}\left(\tau_{1}+\tau_{2} ; \delta, g\right)$ and $\mathrm{M}^{-1}(\tau ; \delta, g)=\mathrm{M}(-\tau ; \delta, g)$. The solution (69) for the Bloch vector in the rotating reference frame is shown graphically in Fig. 1 .

Summarizing these results we can state that the Bloch vector operator and in particular its expectation value, which is an ordinary real-valued three-vector, is performing a precessional motion that is composed of a fast precession about the axis $\mathbf{e}_{3}$ with the angular frequency $\omega_{\mathrm{d}}$ defined by the driving electric quadrupole field and a slow precession (that could also be called a nutation) at the Rabi frequency $\omega_{\mathrm{R}}$ about the direction $\mathbf{e}_{1}^{\prime \prime}=\left(2 g / \omega_{\mathrm{R}}\right) \mathbf{e}_{1}^{\prime}-\left(\delta / \omega_{\mathrm{R}}\right) \mathbf{e}_{3}^{\prime}=\left(2 g / \omega_{\mathrm{R}}\right) \cos \phi_{\mathrm{d}}(t) \mathbf{e}_{1}+\left(2 g / \omega_{\mathrm{R}}\right) \sin \phi_{\mathrm{d}}(t) \mathbf{e}_{2}-$ $\left(\delta / \omega_{\mathrm{R}}\right) \mathbf{e}_{3}$. For general initial values $T_{i}\left(t_{0}\right)$ the time development of the system during the time interval $\tau=t_{1}-t_{0}$ is described by the

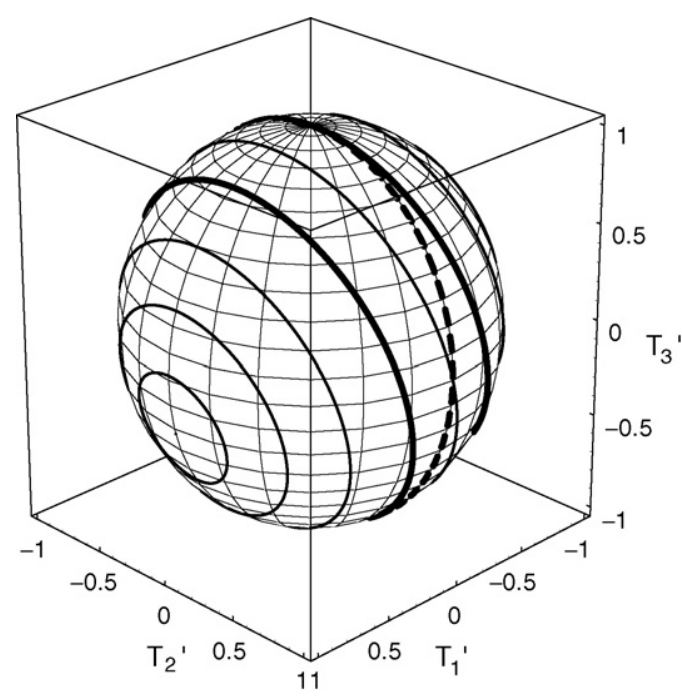

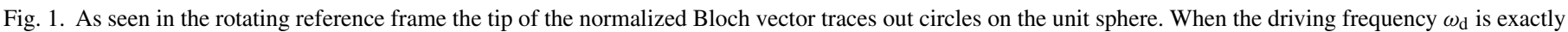

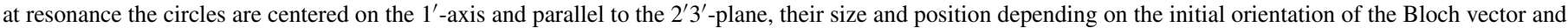

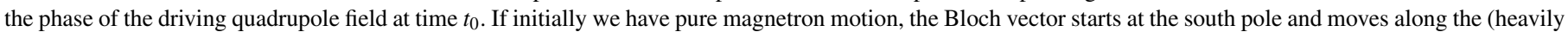

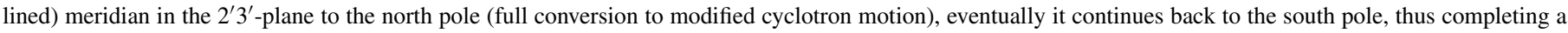

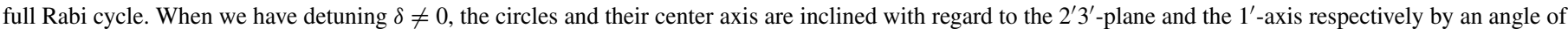

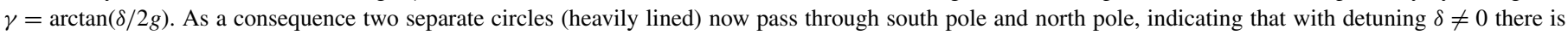

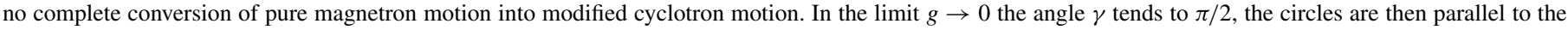
equatorial plane. This case is relevant during the waiting periods in Ramsey excitation. 
Table 1

Comparison between NMR and the Penning trap with quadrupole excitation

\begin{tabular}{lll}
\hline & Nuclear-magnetic-resonance & Penning trap with quadrupole excitation \\
\hline Dynamical variables & Angular momentum $\mathbf{J}$ & Bloch operator $\mathbf{T}$ \\
Dimensionality of Hilbert space & $2 J+1$ & Infinite \\
Static electric field & - & $-\left(U / 2 z_{0}^{2}+r_{0}^{2}\right) \nabla\left(2 z^{2}-x^{2}-y^{2}\right)$ \\
Static magnetic field & $B_{0} \mathbf{e}_{z}$ & $B_{0} \mathbf{e}_{z}$ \\
Rotating electric field & - & $-(2 g \hbar / q) \nabla\left(\cos \phi_{\mathrm{d}}(t) T_{1}+\sin \phi_{\mathrm{d}}(t) T_{2}\right)$ \\
Rotating magnetic field & $B_{1}\left(\mathbf{e}_{1} \cos \omega t-\mathbf{e}_{2} \sin \omega t\right)$ & - \\
Gyromagnetic ratio and analogue & $\gamma=\mu / I$ & $\hbar q / m c$ \\
Larmor frequency and analogue & $\omega_{\mathrm{L}}=\gamma B_{0} / \hbar$ & $\omega_{\mathrm{c}}=q B_{0} / m c$ \\
Hamiltonian & Eq. (74) & Eq. $(61)$ \\
\hline
\end{tabular}

propagator matrix

$$
\mathrm{P}\left(t_{1}, t_{0} ; \delta, g\right)=\mathrm{N}^{-1}\left(\phi_{\mathrm{d}}\left(t_{1}\right)\right) \cdot \mathrm{M}\left(t_{1}-t_{0} ; \delta, g\right) \cdot \mathrm{N}\left(\phi_{\mathrm{d}}\left(t_{0}\right)\right),
$$

where the matrix $\mathrm{N}(t)$ defined in Eq. (66) performs the transition to the rotating frame. The final result can thus be stated in the form

$$
\left(\begin{array}{l}
T_{1}\left(t_{1}\right) \\
T_{2}\left(t_{1}\right) \\
T_{3}\left(t_{1}\right)
\end{array}\right)=\mathrm{P}\left(t_{1}, t_{0} ; \delta, g\right)\left(\begin{array}{l}
T_{1}\left(t_{0}\right) \\
T_{2}\left(t_{0}\right) \\
T_{3}\left(t_{0}\right)
\end{array}\right) .
$$

\subsubsection{Nuclear-magnetic-resonance}

In NMR experiments one studies atomic nuclei with spin $\mathbf{J}=\mathbf{I} \hbar$ and magnetic moment $\vec{\mu}=\gamma \mathbf{I}$, where $\gamma$ is the gyromagnetic ratio. These interact with a strong, constant, homogeneous magnetic field parallel to the three-axis and a weak, homogeneous magnetic field, perpendicular to the three-axis and rotating in the clockwise sense with angular velocity $\omega$ :

$$
\mathbf{B}(t)=B_{1}\left(\mathbf{e}_{1} \cos \omega t-\mathbf{e}_{2} \sin \omega t\right)+B_{0} \mathbf{e}_{3}
$$

In the absence of the rotating field the nuclear spin precesses with the Larmor frequency $\omega_{\mathrm{L}}=\gamma B_{0}$ about the three-axis. The rotating field causes an additional nutational spin motion, which is most pronounced in the resonance case, when $\omega \approx \omega_{\mathrm{L}}$. The energy of the system is

$$
H_{\mathrm{mag}}=-\vec{\mu} \cdot \mathbf{B}=-\hbar \omega_{\mathrm{L}} \cdot I_{3}-\hbar \gamma B_{1}\left(\cos \omega t \cdot I_{1}-\sin \omega t \cdot I_{2}\right)
$$

Considered from the point of view of quantum mechanics, the Hilbert space of the system is $(2 I+1)$-dimensional, the energy levels (Zeeman levels) are equally spaced and separated by an energy $\Delta E=\hbar \omega_{L}$.

A comparison of the similarities and dissimilarities of the two dynamical systems, nuclear-magnetic-resonance and the ion motion in a Penning trap with quadrupole excitation, is found in Table 1.

In nuclear-magnetic-resonance one deals with a particle endowed with a magnetic moment interacting with homogeneous magnetic fields, the dynamic variables, i.e., the components of the spin angular momentum vector describe an intrinsic property of the particle. On the other hand, studying the motion of an ion in a Penning trap with quadrupole excitation, one deals with a scalar particle with electric charge, moving through inhomogeneous electric fields. The vector operator in this case, i.e., the Bloch vector, does not describe an intrinsic property of the particle, but is a quantity derived from the basic dynamical variables $a_{+}, a_{-}, a_{+}^{\dagger}, a_{+}^{\dagger}$, which describe the spatial motion of the particle. As a derived quantity the Bloch vector operator describes only special aspects of the ion motion, not the problem in its full generality. The common features of both systems are that the dynamical variables are the components of a vector operator with $S U(2)$ commutation relations, that the Hamiltonian is linear in these dynamical variables and that (apart from trivial signs) the explicit time dependence of the Hamiltonian is the same for both dynamical systems. The fact that the ion motion in the Penning trap with quadrupole excitation requires an infinite-dimensional Hilbert space is of no real consequence, because this Hilbert space decomposes into finite-dimensional subspaces that are invariant under the quadrupole interaction. This interaction preserves the total number of quanta in the system, $N_{\text {tot }}=2 T_{0}$, so that $T_{0}$ commutes with the Hamiltonian and can be treated like a constant. It is therefore justified to view nuclear-magnetic-resonance and the motion of the Bloch vector associated with the ion motion in a Penning trap as two different physical realizations of the same abstract dynamical system, defined in terms of a vector operator with $S U(2)$ commutation relations and a time-dependent Hamiltonian linear in the components of the vector operator. 


\section{Ramsey excitation}

In his Nobel lecture [4] Ramsey introduced the "method of separated oscillatory fields" by the example of nuclear-magneticresonance. We have shown in the preceding section that the interconversion of two motional modes in a Penning trap by resonant electric quadrupole radiation and the change of orientation of a nuclear spin by a magnetic field rotating at the Larmor frequency are just two different physical realizations of the same abstract dynamical system. Therefore, in studying the Ramsey method we may expect to find many close analogies between our work with Penning traps and applications of this method to magnetic resonance.

\subsection{Basic considerations}

In mass spectrometry with Penning traps the crucial experimental step is the preparation of an ion sample in a state of pure magnetron motion in a precision Penning trap, the subsequent conversion of the magnetron mode into the modified cyclotron mode by interaction with resonant electric quadrupole radiation at the true cyclotron frequency $\omega_{\mathrm{c}}$, and finally the measurement of the amount of energy residing in the modified cyclotron mode by a time-of-flight detection technique. This experimental method has been first described in seminal papers by Bollen et al. [5] and König et al. [20]. These authors have also compared their experimental data to a detailed theoretical analysis of the expected resonance line shapes that they had developed on the basis of the classical ion trajectory concept. The present paper uses a different conceptual framework based in quantum mechanics, confirming in the end the earlier results. The 'resonance lineshapes' calculated in our approach represent the probability for conversion of a state of initially pure magnetron motion into cyclotron motion for given values of the duration $\tau$ of the quadrupolar rf-pulse and the detuning $\delta$. In other words, they indicate the percentage of the number of initially present magnetron quanta that has been converted into cyclotron quanta. Since $\omega_{-} \ll \omega_{+}$the radial ion energy after the excitation effectively measures the number of cyclotron quanta created in the conversion process. Before a comparison to experimental data can be made the modification of the curves due to the final time-of-flight measurement [7] must still be folded in, as discussed in the accompanying paper by George et al. [1].

The preparation of the ion sample in a state of pure magnetron motion defines the initial state of our system. In more formal terms, on the basis of our actual knowledge or our assumptions about the initial state of the system at time $t_{0}$ we must calculate the expectation values of the components of the Bloch operator $\mathbf{T}\left(t_{0}\right)$ and of the time independent operator $T_{0}$ as input into the calculation. The density matrix formalism provides a general framework for this purpose. It allows to introduce statistical assumptions where we lack precise knowledge, for example about initial phases. With the density operator denoted by $\rho$ we then obtain the expectation values

$$
\left\langle T_{0}\right\rangle=\operatorname{Tr}\left\{\rho T_{0}\right\}, \quad\left\langle T_{1}\left(t_{0}\right)\right\rangle=\operatorname{Tr}\left\{\rho T_{1}\left(t_{0}\right)\right\}, \quad\left\langle T_{2}\left(t_{0}\right)\right\rangle=\operatorname{Tr}\left\{\rho T_{2}\left(t_{0}\right)\right\}, \quad\left\langle T_{3}\left(t_{0}\right)\right\rangle=\operatorname{Tr}\left\{\rho T_{3}\left(t_{0}\right)\right\} .
$$

This means that initial situations are characterized by four real numbers, in particular, the expectation value of the Bloch vector operator is an ordinary real-valued three-vector. The simplest choice of initial values and at the same time the one of greatest interest is represented by

$$
\left\langle T_{0}\right\rangle=-\left\langle T_{3}\left(t_{0}\right)\right\rangle \neq 0, \quad\left\langle T_{1}\left(t_{0}\right)\right\rangle=\left\langle T_{2}\left(t_{0}\right)\right\rangle=0 .
$$

It implies the presence of $\left\langle N_{\text {tot }}\right\rangle=2\left\langle T_{0}\right\rangle$ quanta in the system, all of them in magnetron mode, because $\left\langle N_{-}\left(t_{0}\right)\right\rangle=\left\langle T_{0}-T_{3}\left(t_{0}\right)\right\rangle$ and $\left\langle N_{+}\left(t_{0}\right)\right\rangle=\left\langle T_{0}+T_{3}\left(t_{0}\right)\right\rangle$. The experiment converts quanta in the magnetron mode into quanta in the modified cyclotron mode, so that at the final time $t_{1}$ we have $\left\langle N_{+}\left(t_{1}\right)\right\rangle=\left\langle T_{0}+T_{3}\left(t_{1}\right)\right\rangle$ and $\left\langle N_{-}\left(t_{1}\right)\right\rangle=\left\langle T_{0}-T_{3}\left(t_{1}\right)\right\rangle$. In the case of complete conversion $\left\langle N_{+}\left(t_{1}\right)\right\rangle=\left\langle N_{\text {tot }}\right\rangle$ and $\left\langle N_{-}\left(t_{1}\right)\right\rangle=0$. The quantity that is measured in the experiment is the amount of energy transferred to the modified cyclotron mode $\left\langle E_{+}\left(t_{1}\right)\right\rangle=\hbar \omega_{+}\left\langle N_{+}\left(t_{1}\right)\right\rangle$. When the initial state is a pure quantum state $|\psi\rangle$ then the density matrix takes the simple form $\rho=|\psi\rangle\langle\psi|$ and expectation values are simply given by $\left\langle T_{i}\left(t_{0}\right)\right\rangle=\left\langle\psi\left|T_{i}\left(t_{0}\right)\right| \psi\right\rangle$. This has the consequence that for example the expectation value of the operator $N_{+}(t)$ may be written as the square of the norm of a Hilbert space vector: $\left\langle N_{+}(t)\right\rangle=\left\langle\psi\left|a_{+}^{\dagger}(t) a_{+}(t)\right| \psi\right\rangle=\| a_{+}(t)|\psi\rangle \|^{2}$. We shall exploit this fact in the next subsection for the convenient calculation of the Ramsey profil functions.

As an example for the general initial value problem let us consider a single ion in a quasiclassical quantum state, a so called coherent oscillator state. Ignoring the axial oscillator, such a quantum state is defined by two complex numbers, $\alpha_{+}=\left|\alpha_{+}\right| \mathrm{e}^{-\mathrm{i} \chi_{+}}$and $\alpha_{-}=\left|\alpha_{-}\right| \mathrm{e}^{\mathrm{i} \chi_{-}}$, that chararacterize the modified cyclotron motion and the magnetron motion, respectively. We write $|\psi\rangle=\left|\alpha_{+}, \alpha_{-}\right\rangle$. Using the defining property of coherent states, $a_{ \pm}|\psi\rangle=\alpha_{ \pm}|\psi\rangle$, we can easily compute the expectation values of $T_{0}$ and $\mathbf{T}$,

$$
\begin{aligned}
& \left\langle T_{0}\right\rangle=\frac{1}{2}\left(\left|\alpha_{+}\right|^{2}+\left|\alpha_{-}\right|^{2}\right), \quad\left\langle T_{1}\right\rangle=\left|\alpha_{+}\right| \cdot\left|\alpha_{-}\right| \cos \left(\chi_{+}+\chi_{-}\right), \\
& \left\langle T_{2}\right\rangle=\left|\alpha_{+}\right| \cdot\left|\alpha_{-}\right| \sin \left(\chi_{+}+\chi_{-}\right), \quad\left\langle T_{3}\right\rangle=\frac{1}{2}\left(\left|\alpha_{+}\right|^{2}-\left|\alpha_{-}\right|^{2}\right) .
\end{aligned}
$$

This result shows that for the classical trajectory of a single ion with precisely known initial values the conversion process will depend on the phases of the magnetron and the modified cyclotron oscillators. Phase independent results are obtained only for the special cases of initially pure magnetron motion $\left(\left|\alpha_{+}\right|=0\right)$ or initially pure modified cyclotron motion $\left(\left|\alpha_{-}\right|=0\right)$. In practice, 
however, our knowledge of the initial phases is often incomplete. It is then necessary to make up for this lack of information by statistical assumptions, in other words to work with density matrices. For example, in the extreme case of no phase information at all one would assume all values of the two phases to be equally probable and postulate a density matrix

$$
\rho=\frac{1}{(2 \pi)^{2}} \int_{0}^{2 \pi} \mathrm{d} \chi_{+} \int_{0}^{2 \pi} \mathrm{d} \chi_{-}\left|\alpha_{+}, \alpha_{-}\right\rangle\left\langle\alpha_{-}, \alpha_{+}\right| .
$$

The expectation values of $T_{0}$ and the initial Bloch vector components then become

$$
\left\langle T_{i}\right\rangle=\operatorname{Tr}\left\{\rho T_{i}\right\}=\frac{1}{(2 \pi)^{2}} \int_{0}^{2 \pi} \mathrm{d} \chi_{+} \int_{0}^{2 \pi} \mathrm{d} \chi_{-}\left\langle\alpha_{-}, \alpha_{+}\left|T_{i}\right| \alpha_{+}, \alpha_{-}\right\rangle,
$$

that is $\left\langle T_{0}\right\rangle=(1 / 2)\left(\left|\alpha_{+}\right|^{2}+\left|\alpha_{-}\right|^{2}\right),\left\langle T_{1}\right\rangle=\left\langle T_{2}\right\rangle=0$, and $\left\langle T_{3}\right\rangle=(1 / 2)\left(\left|\alpha_{+}\right|^{2}-\left|\alpha_{-}\right|^{2}\right)$.

\subsection{The one-pulse excitation scheme}

In this section we study the standard excitation scheme, where the ions in the Penning trap are acted upon by a single pulse of electric quadrupole radiation of frequency $\omega_{\mathrm{d}}$, with amplitude $g$, and of duration $\tau$. Initially the ions are prepared in a state of pure magnetron motion, the aim is to convert their motional mode as completely as possible into modified cyclotron motion, since maximal conversion is the signature for the resonance frequency $\omega_{\mathrm{c}}$. This process depends on $g, \tau$, and the detuning $\delta=\omega_{\mathrm{d}}-\omega_{\mathrm{c}}$. Our theoretical approach is based on following the time development of the expectation value of the Bloch vector operator over the time interval $\tau$. An ion in a state of pure magnetron motion at the initial time $t_{0}$ requires the initial condition (75). This condition tells us that the state of the ion is built from $\left\langle N_{\text {tot }}\right\rangle=\left\langle N_{-}\right\rangle=\left\langle T_{0}-T_{3}\left(t_{0}\right)\right\rangle$ excitation quanta residing in the magnetron oscillator. While the pulse of electric quadrupole radiation acts on the ion the expectation value of the Bloch vector operator performs its precessional and nutational motion according to Eq. (72). After the pulse has terminated at time $t_{1}=t_{0}+\tau$ the expected numbers of modified cyclotron and magnetron quanta are given by $\left\langle N_{+}\left(t_{1}\right)\right\rangle=\left\langle T_{0}+T_{3}\left(t_{1}\right)\right\rangle$ and $\left\langle N_{-}\left(t_{1}\right)\right\rangle=\left\langle T_{0}-T_{3}\left(t_{1}\right)\right\rangle$, respectively. Observing that $\left\langle T_{0}\right\rangle=\left\langle N_{\text {tot }} / 2\right\rangle=-\left\langle T_{3}\left(t_{0}\right)\right\rangle$ we can formulate the normalized result for the excitation of the modified cyclotron motion for general $\delta, \tau$, and $g$ in terms of the one-pulse profil function:

$$
F_{1}(\delta ; \tau, g)=\left\langle N_{+}\left(t_{0}+\tau\right)\right\rangle /\left\langle N_{\text {tot }}\right\rangle=\frac{1}{2}\left[1+\left\langle T_{3}\left(t_{0}+\tau\right)\right\rangle /\left\langle T_{0}\right\rangle\right] .
$$

Inserting the results from Eqs. (71) and (72) we find that the explicit expression for $F_{1}$ is given by the 33-element of the matrix $\mathrm{M}(\tau ; \delta, g)$, Eq. (70),

$$
F_{1}(\delta ; \tau, g)=\frac{1}{2}\left(1-\mathrm{M}_{33}\right)=-\frac{4 g^{2}}{2 \omega_{\mathrm{R}}^{2}}\left(\cos \omega_{\mathrm{R}} \tau-1\right)=\frac{4 g^{2}}{\omega_{\mathrm{R}}^{2}} \sin ^{2}\left(\frac{\omega_{\mathrm{R}} \tau}{2}\right) .
$$

The same expression would have been obtained by assuming the ion at time $t_{0}$ to be in a pure quantum state $|\psi\rangle$, using Eqs. (41) and (42), and arguing that

$$
\begin{aligned}
\left\langle N_{+}\left(t_{0}+\tau\right)\right\rangle & =\left\langle\psi\left|a_{+}^{\dagger}\left(t_{0}+\tau\right) a_{+}\left(t_{0}+\tau\right)\right| \psi\right\rangle=\| a_{+}\left(t_{0}+\tau\right)|\psi\rangle\left\|^{2}=\frac{4 g^{2}}{\omega_{\mathrm{R}}^{2}} \sin ^{2}\left(\omega_{\mathrm{R}} \tau\right) \cdot\right\| a_{-}\left(t_{0}\right)|\psi\rangle \|^{2} \\
& =\frac{4 g^{2}}{\omega_{\mathrm{R}}^{2}} \sin ^{2}\left(\frac{\omega_{\mathrm{R}} \tau}{2}\right) \cdot\left\langle N_{\mathrm{tot}}\right\rangle .
\end{aligned}
$$

Exactly on resonance $(\delta=0)$ the one-pulse profil reduces to $F_{1}(\delta=0 ; \tau, g)=\sin ^{2} g \tau$. This tells us that the initially pure magnetron motion has been transformed completely into pure modified cyclotron motion after the conversion time $\tau_{\mathrm{c}}=\pi / 2 g$. If the pulse of quadrupole radiation acts for a time longer than $\tau_{\mathrm{c}}$ the transformation is reversed until for $\tau=2 \tau_{\mathrm{c}}$ we again have pure magnetron motion. This transformation continues in a periodic fashion.

It is evident that the conversion time $\tau_{\mathrm{c}}=\pi / 2 \mathrm{~g}$ represents a natural time unit for our study. Thus introducing the dimensionless variables $\theta_{1}=\tau / \tau_{\mathrm{c}}$ and $\eta=\delta / 2 g$ to describe duration and detuning of a pulse of quadrupole radiation, the result of the conversion process as a function of $\theta_{1}$ and $\eta$ can be visualized by a universal profile function

$$
f_{1}\left(\eta ; \theta_{1}\right)=\frac{1}{1+\eta^{2}} \cdot \sin ^{2}\left(\frac{\pi}{2} \theta_{1} \sqrt{1+\eta^{2}}\right),
$$

which is equivalent to Eq. (81). Fig. 2 shows a three-dimensional plot of this function. A section through this plot corresponding to a plane $\theta_{1}=$ const produces the line profile as a function of detuning, or more precisely of $\eta=\delta / 2 g$, to be expected in an experiment. As an example we show in Fig. 3 the line shapes to be expected after pulses of duration $\tau=\tau_{\mathrm{c}}\left(\theta_{1}=1\right.$, solid line) and $\tau=3 \tau_{\mathrm{c}}$ $\left(\theta_{1}=3\right.$, dashed line $)$. 


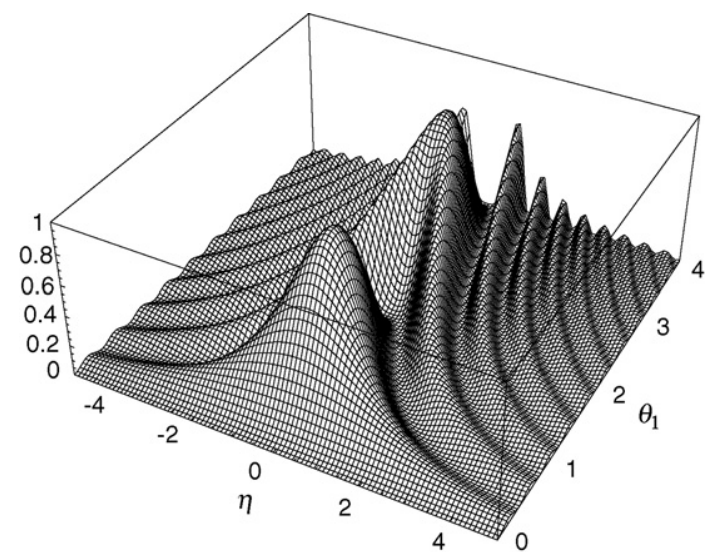

Fig. 2. Landscape profile showing the probability for conversion of pure magnetron motion into modified cyclotron motion as a function of the dimensionless variables $\theta_{1}$ (pulse duration in units of conversion time $\tau_{\mathrm{c}}$ ) and $\eta=\delta / 2 g$.

The width of the central peak is relevant for the precision of the mass measurement. It is determined by the position of the two zeroes next to $\eta=0$. It is easily seen from Eq. (83) that after a pulse of duration $\tau=(2 n+1) \tau_{\mathrm{c}}$ (i.e., $n+\frac{1}{2}$ complete Rabi cycles) these zeroes are at $\eta_{0}=\sqrt{4 n+3} /(2 n+1)$ (for example see Fig. 3: $\eta_{0}=\sqrt{3}=1.732$ for $n=0$ and $\eta_{0}=\sqrt{7} / 3=0.882$ for $n=1$ ). The value of $\eta_{0}$ roughly equals the full width of the central peak at half maximum. The relation $\eta=\delta / 2 g=\delta \cdot \tau_{\mathrm{c}} / \pi$ implies that in terms of frequencies the detuning $\delta_{0}$ at the position of the first zero obeys a sort of time-energy uncertainty relation: $\hbar \delta_{0} \cdot(2 n+1) \tau_{\mathrm{c}}=h \sqrt{n+0.75}$. The width of the central peak shrinks with decreasing amplitude $g$ of the quadrupole radiation, inversely proportional to the conversion time $\tau_{\mathrm{c}}$.

Finally we compare the profile function of Eq. (83) to the Fourier transform of a scalar wave train $u(t)=g \exp \left[-i\left(\omega_{0} t+\right.\right.$ $\chi)]$ of finite duration $\tau$. Defining $\delta=\omega-\omega_{0}, \eta=\delta / 2 g, \tau_{\mathrm{c}}=\pi / 2 g$, and $\theta_{1}=\tau / \tau_{\mathrm{c}}$, the Fourier transform becomes $\tilde{u}(\omega)=$ $\int_{-\tau / 2}^{+\tau / 2} \mathrm{~d} t \mathrm{e}^{\mathrm{i} \omega t} u(t)=\mathrm{e}^{-\mathrm{i} \chi} \sin \left(\pi \theta_{1} \eta / 2\right) / \eta$. For the intensity of the wave we obtain $I(\omega)=|\tilde{u}(\omega)|^{2}=\sin ^{2}\left(\pi \theta_{1} \eta / 2\right) / \eta^{2}$. This result closely resembles Eq. (83), in fact it could be obtained from Eq. (83) by the substitution $\sqrt{1+\eta^{2}} \rightarrow \eta$. This means that with respect to the variable $\eta$ the line profile for the quadrupole excitation of the ion motion is more narrow than that for the Fourier transform of a scalar wave (zeroes of the central peak at $\eta_{0}= \pm 1.732 \cdot \theta_{1}^{-1}$ as compared to $\eta_{0}= \pm 2 \cdot \theta_{1}^{-1}$ ).

\subsection{The two-pulse excitation scheme}

The excitation of a physical system by two time separated pulses of radiation with a well-defined phase relation is the central idea of Ramsey's method. To understand qualitatively what kind of result we may expect from this method we first recall the well-known experiment of diffraction of a plane scalar wave $u$ from a double slit. Denote the width of each of the two slits by $a$, the distance between the two slits by $d$, the wavelength of the scalar wave by $\lambda$, then the intensity of the diffracted wave on a distant screen is found to be $I=|u|^{2} \propto 4 \cos ^{2}[(\pi(a+d) / \lambda) \sin \alpha] \sin ^{2}[(\pi a / \lambda) \sin \alpha] / \sin ^{2} \alpha$, where $\alpha$ is the diffraction angle. The diffraction pattern consists of a number of intensity peaks separated by the 1 st class minima at $\sin \alpha= \pm n \lambda / a(n=1,2,3, \ldots)$ and the second class minima at $\sin \alpha= \pm n \lambda / 2(a+d)(n=1,3,5, \ldots)$. The width of the central diffraction peak is determined by its zeroes at $\sin \alpha= \pm \lambda / 2(a+d)$. This relation can be read as a position-momentum uncertainty relation $(a+d) \cdot p_{x}=h / 2$, where $p_{x}=\sin \alpha \cdot h / \lambda$ is the lateral momentum of a diffracted particle. With increasing separation $d$ of the slits the position uncertainty

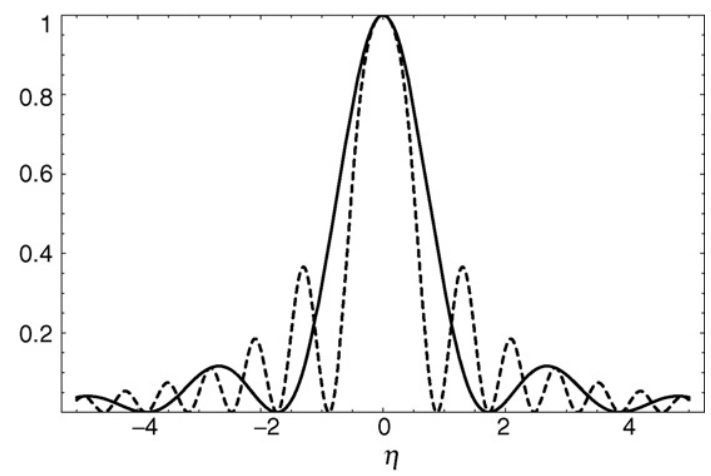

Fig. 3. Line shape for complete conversion of pure magnetron motion into modified cyclotron motion for pulse duration $\tau=\tau_{\mathrm{c}}$ (solid line) and $\tau=3 \tau_{\mathrm{c}}$ (dashed line). 
of the particle increases, at the same time the momentum uncertainty decreases, which is reflected in the decreasing width of the central diffraction peak. For more easy comparison with the analysis in the timelike domain we now change the notation to $\eta=\sin \alpha$, $\theta_{1}=2 a / \lambda$, and $\theta_{0}=2 d / \lambda$. With suitable normalization of the incoming plane wave the intensity of the wave diffracted from the double slit is then given by

$$
I\left(\eta ; \theta_{0}, \theta_{1}\right)=\frac{4}{\eta^{2}} \sin ^{2}\left(\frac{\pi}{2} \theta_{1} \eta\right) \cos ^{2}\left(\frac{\pi}{2}\left(\theta_{0}+\theta_{1}\right) \eta\right) .
$$

Consider next the timelike analogue to the double slit experiment, namely the Fourier transform with respect to time of a sequence of two wave trains of a scalar wave with circular frequency $\omega_{0}$ and with a well-defined phase relation, $u(t)=g \exp \left[-i\left(\omega_{0} t+\chi_{1}\right)\right]$ for $-\left(\tau_{1}+\tau_{0} / 2\right) \leq t \leq-\tau_{0} / 2$ and $u(t)=g \exp \left[-i\left(\omega_{0} t+\chi_{2}\right)\right]$ for $\tau_{0} / 2 \leq t \leq \tau_{0} / 2+\tau_{1}, u(t)=0$ otherwise. With $\delta=\omega-\omega_{0}$ and $\Delta \chi=\chi_{1}-\chi_{2}$ the Fourier transform $\tilde{u}(\omega)=\int_{-\infty}^{+\infty} \mathrm{d} t \mathrm{e}^{\mathrm{i} \omega t} u(t)$ yields for the intensity $I=\tilde{u}^{2}$ the expression

$$
I\left(\eta ; \tau_{0}, \tau_{1}\right)=\frac{16 g^{2}}{\delta^{2}} \sin ^{2}\left(\delta \tau_{1} / 2\right) \cos ^{2}\left(\delta\left(\tau_{0}+\tau_{1}\right) / 2+\Delta \chi / 2\right) .
$$

Introducing the time unit $\tau_{\mathrm{c}}=\pi / 2 g$ and the dimensionless variables $\eta=\delta / 2 g, \theta_{0}=\tau_{0} / \tau_{\mathrm{c}}$, and $\theta_{1}=\tau_{1} / \tau_{\mathrm{c}}$ the intensity is given by Eq. (84) above, i.e., the expression is exactly analoguous to the result of the doubleslit experiment. It can therefore be interpreted in a similar fashion in terms of a time-energy uncertainty relation. Eq. (84) describes a pattern of peaks, separated by the first class and second class zeroes. In particular, the central peak is limited by the zeroes at $\eta_{0}=\left(\theta_{0}+\theta_{1}\right)^{-1}$.

Keeping in mind these preparatory remarks we now turn to the discussion of the two-pulse excitation scheme for ion motion in the Penning trap. The most general and also the conceptually most transparent approach is again based on the concept of the Bloch vector operator and its expectation value. The simplest possible Ramsey excitation scheme uses two pulses of quadrupole radiation with equal phase $\phi_{\mathrm{d}}(t)$, of equal duration $\tau_{1}$ and equal amplitude $g$, separated by a waiting time interval $\tau_{0}$. We treat the simplest possible initial condition, Eq. (75), assuming that at time $t_{0}$ all oscillator quanta are in the magnetron mode. All we have to do is to follow the time development of the expectation value of the three-component of the Bloch vector operator during the time interval of interaction between ion and quadrupole radiation, $t_{0} \leq t \leq t_{1}=t_{0}+\tau_{0}+2 \tau_{1}$, using our previous result Eq. (72). The quantity of interest to us is the ratio of the expectation value of the number of quanta in the modified cyclotron oscillator after the excitation process has been completed, $\left\langle N_{+}\left(t_{1}\right)\right\rangle=\left\langle T_{0}\right\rangle+\left\langle T_{3}\left(t_{1}\right)\right\rangle$, to the total number of quanta present in the system, $\left\langle N_{\text {tot }}\right\rangle=2\left\langle N_{0}\right\rangle=\left\langle N_{-}\left(t_{0}\right)\right\rangle$, as a function of $\delta, \tau_{0}, \tau_{1}$, and $g$. This general function shall be described as the two-pulse profile function

$$
F_{2}\left(\delta ; \tau_{0}, \tau_{1}, g\right)=\left\langle N_{+}\left(t_{0}+\tau_{0}+2 \tau_{1}\right)\right\rangle /\left\langle N_{\text {tot }}\right\rangle=\frac{1}{2}\left[1+\left\langle T_{3}\left(t_{0}+\tau_{0}+2 \tau_{1}\right)\right\rangle /\left\langle T_{0}\right\rangle\right]
$$

In order to calculate this function we start with the general solution for the time development of the Bloch vector operator, Eq. (72), or more precisely, with its expectation value

$$
\left(\begin{array}{c}
\left\langle T_{1}\left(t_{3}\right)\right\rangle \\
\left\langle T_{2}\left(t_{3}\right)\right\rangle \\
\left\langle T_{3}\left(t_{3}\right)\right\rangle
\end{array}\right)=\mathrm{P}\left(t_{3}, t_{2} ; \delta, g\right) \cdot \mathrm{P}\left(t_{2}, t_{1} ; \delta, 0\right) \cdot \mathrm{P}\left(t_{1}, t_{0} ; \delta, g\right)\left(\begin{array}{c}
\left\langle T_{1}\left(t_{0}\right)\right\rangle \\
\left\langle T_{2}\left(t_{0}\right)\right\rangle \\
\left\langle T_{3}\left(t_{0}\right)\right\rangle
\end{array}\right),
$$

with $t_{1}=t_{0}+\tau_{1}, t_{2}=t_{1}+\tau_{0}=t_{0}+\tau_{1}+\tau_{0}, t_{3}=t_{2}+\tau_{1}=t_{0}+2 \tau_{1}+\tau_{0}$. On account of Eq. (71) the product of the three propagator matrices $\mathrm{P}$ can be reduced to

$$
\mathrm{P}\left(t_{3}, t_{2} ; \delta, g\right) \cdot \mathrm{P}\left(t_{2}, t_{1} ; \delta, 0\right) \cdot \mathrm{P}\left(t_{1}, t_{0} ; \delta, g\right)=\mathrm{N}^{-1}\left(\phi_{\mathrm{d}}\left(t_{3}\right)\right) \cdot \mathrm{R}^{(2)}\left(\delta ; \tau_{0}, \tau_{1}, g\right) \cdot \mathrm{N}\left(\phi_{\mathrm{d}}\left(t_{0}\right)\right) .
$$

Here we have introduced the two-pulse Ramsey matrix $\mathrm{R}^{(2)}\left(\delta ; \tau_{0}, \tau_{1}, g\right)$, defined as a product of propagation matrices $\mathrm{M}\left(\tau_{1} ; \delta, g\right)$ (see Eq. (70))

$$
\mathrm{R}^{(2)}\left(\delta ; \tau_{0}, \tau_{1}, g\right)=\mathrm{M}\left(\tau_{1} ; \delta, g\right) \cdot \mathrm{M}\left(\tau_{0} ; \delta, 0\right) \cdot \mathrm{M}\left(\tau_{1} ; \delta, g\right)
$$

To calculate the two-pulse profil function we need only the three-component $T_{3}\left(t_{0}+\tau_{0}+2 \tau_{1}\right)$, moreover the initial condition Eq. (75) tells us that $\left\langle T_{1}\left(t_{0}\right)\right\rangle=\left\langle T_{2}\left(t_{0}\right)\right\rangle=0$. Therefore it is sufficient to find the detailed expression for $\mathrm{R}_{33}^{(2)}\left(\delta ; \tau_{0}, \tau_{1}, g\right)$. Using Eq. $(70)$ one finds after laborious algebra the following result for the two-pulse profile function (Fig. 4)

$$
\begin{aligned}
F_{2}\left(\delta ; \tau_{0}, \tau_{1}, g\right) & =\frac{1}{2}\left(1-\mathrm{R}_{33}^{(2)}\right)=\frac{4 g^{2}}{\omega_{\mathrm{R}}^{2}}\left\{\cos \left(\frac{\delta \tau_{0}}{2}\right) \sin \left(\omega_{\mathrm{R}} \tau_{1}\right)+\frac{\delta}{\omega_{\mathrm{R}}} \sin \left(\frac{\delta \tau_{0}}{2}\right)\left[\cos \left(\omega_{\mathrm{R}} \tau_{1}\right)-1\right]\right\}^{2} \\
& =\frac{16 g^{2}}{\omega_{\mathrm{R}}^{2}} \sin ^{2}\left(\frac{\omega_{\mathrm{R}} \tau_{1}}{2}\right) \cdot\left\{\cos \left(\frac{\delta \tau_{0}}{2}\right) \cos \left(\frac{\omega_{\mathrm{R}} \tau_{1}}{2}\right)-\frac{\delta}{\omega_{\mathrm{R}}} \sin \left(\frac{\delta \tau_{0}}{2}\right) \sin \left(\frac{\omega_{\mathrm{R}} \tau_{1}}{2}\right)\right\}^{2} .
\end{aligned}
$$




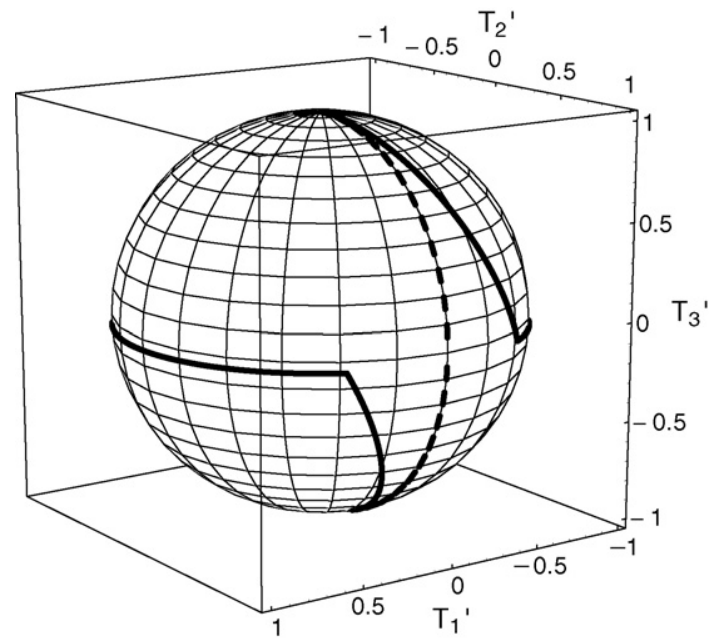

Fig. 4. Typical paths on the unit sphere traced out by the tip of the normalized Bloch vector during Ramsey excitation (heavy lines), assuming that the ions are initially in a state of pure magnetron motion and hence the paths start out at the south pole. In the case of exact resonance the tip of the Bloch vector moves during the first excitation pulse of duration $\tau_{1}=\tau_{\mathrm{c}} / 2$ along the meridian from the south pole to the equator, during the waiting time $\tau_{0}$ it stays at rest, during the second excitation pulse $\tau_{1}$ it continues to move along the meridian from the equator to the north pole, where we have achieved complete conversion to modified cyclotron motion. On the other hand, for detuning $\delta \neq 0$ the tip of the Bloch vector moves during the first excitation pulse $\tau_{1}$ from the south pole to some point south of the equator along a circular arc, which is inclined with regard to the $2^{\prime} 3^{\prime}$-plane by an angle of $\gamma=\arctan (\delta / 2 g)$, during the waiting period $\tau_{0}$ it continues along a parallel to the equator for an arc distance $\propto \delta \tau_{0}$, during the second excitation pulse $\tau_{1}$ it moves again along an inclined circular arc. The end point reached at the completion of Ramsey excitation scheme indicates what degree of conversion of magnetron into modified cyclotron motion has been achieved by this procedure.

It is interesting to note that in its second formulation this result (after appropriate matching of the notation) corresponds exactly to Eq. (12) in Ramsey's paper [3], where the method of separated oscillating fields is discussed in the original context of molecular beams passing through magnetic fields.

As in the discussion of one-pulse quadrupole excitation an alternative way of reasoning exists, starting from the assumption that in the Heisenberg picture our physical system is characterized by a state vector $|\psi\rangle$, and observing that after the two-pulse Ramsey excitation the expectation value for the number of modified cyclotron quanta is

$$
\left\langle N_{+}\left(t_{0}+\tau_{0}+2 \tau_{1}\right)\right\rangle=\left\langle\psi\left|a_{+}^{\dagger}\left(t_{0}+\tau_{0}+2 \tau_{1}\right) a_{+}\left(t_{0}+\tau_{0}+2 \tau_{1}\right)\right| \psi\right\rangle=\| a_{+}\left(t_{0}+\tau_{0}+2 \tau_{1}\right)|\psi\rangle \|^{2} .
$$

Here we have to insert our results on the time development of the modal annihilation and creation operators, Eq. (42), formulated in terms of complex $2 \times 2$ matrices. Again it is convenient to introduce a Ramsey matrix

$$
\mathcal{R}^{(2)}\left(\delta ; \tau_{0}, \tau_{1}, g\right)=\mathcal{M}\left(\tau_{1} ; \delta, g\right) \cdot \mathcal{M}\left(\tau_{0} ; \delta, 0\right) \cdot \mathcal{M}\left(\tau_{1} ; \delta, g\right) .
$$

The initial condition Eq. (75) implies $a_{+}\left(t_{0}\right)|\psi\rangle=0$, so that only the 12-element of the Ramsey matrix needs to be calculated. We obtain

$$
a_{+}\left(t_{0}+\tau_{0}+2 \tau_{1}\right)|\psi\rangle=\exp \left[-i\left(\omega_{+}+\delta / 2\right)\left(\tau_{0}+2 \tau_{1}\right)-\frac{i}{2} \phi_{\mathrm{d}}\left(t_{0}\right)\right] \cdot \mathcal{R}_{12}^{(2)}\left(\delta ; \tau_{0}, \tau_{1}, g\right) \cdot a_{-}\left(t_{0}\right)|\psi\rangle .
$$

The square of the norm of this equation becomes

$$
\left\langle N_{+}\left(t_{0}+\tau_{0}+2 \tau_{1}\right)\right\rangle=\|\left. a_{+}\left(t_{0}+\tau_{0}+2 \tau_{1}\right)|\psi\rangle\right|^{2}=\left|\mathcal{R}_{12}^{(2)}\left(\delta ; \tau_{0}, \tau_{1}, g\right)\right|^{2} \cdot\left\langle N_{-}\left(t_{0}\right)\right\rangle .
$$

Remembering that $\left\langle N_{-}\left(t_{0}\right)\right\rangle=\left\langle N_{\text {tot }}\right\rangle=2\left\langle N_{0}\right\rangle$ the two-pulse profil function can now be written as

$$
F_{2}\left(\delta ; \tau_{0}, \tau_{1}, g\right)=\left\langle N_{+}\left(t_{0}+\tau_{0}+2 \tau_{1}\right)\right\rangle /\left\langle N_{\text {tot }}\right\rangle=\left|\mathcal{R}_{12}^{(2)}\left(\delta ; \tau_{0}, \tau_{1}, g\right)\right|^{2} .
$$

The matrix element $\mathcal{R}_{12}^{(2)}$ must now be calculated using the complex $2 \times 2$ matrix $\mathcal{M}$ in Eq. (41). This approach leads to the same result (91) as the previous one, but it has the advantage that it yields directly an expression of the form $\left|\mathcal{R}_{12}^{(2)}\left(\delta ; \tau_{0}, \tau_{1}, g\right)\right|^{2}$.

The two-pulse profile function $F_{2}\left(\delta ; \tau_{0}, \tau_{1}, g\right)$ depends on four variables. For a study of its general properties it is advantageous to use the conversion time $\tau_{\mathrm{c}}=\pi / 2 g$ as a time unit and transform the profile function to the dimensionless variables $\eta=\delta / 2 g$, $\theta_{0}=\tau_{0} / \tau_{\mathrm{c}}$, and $\theta_{1}=\tau_{1} / \tau_{\mathrm{c}}$. Eq. (91) is then equivalent to a function of only three independent variables,

$$
f_{2}\left(\eta ; \theta_{0}, \theta_{1}\right)=\frac{1}{1+\eta^{2}} \cdot\left\{\cos \left(\frac{\pi}{2} \theta_{0} \eta\right) \cdot \sin \left(\pi \theta_{1} \sqrt{1+\eta^{2}}\right)+\frac{\eta}{\sqrt{1+\eta^{2}}} \cdot \sin \left(\frac{\pi}{2} \theta_{0} \eta\right) \cdot\left[\cos \left(\pi \theta_{1} \sqrt{1+\eta^{2}}\right)-1\right]\right\}^{2} .
$$




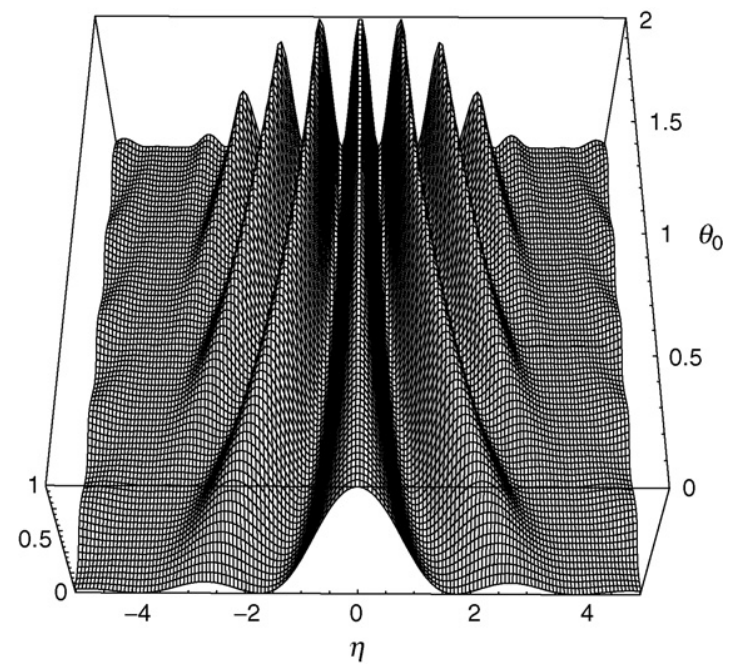

Fig. 5. Landscape profile for two-pulse Ramsey excitation showing the probability for the conversion of pure magnetron motion into modified cyclotron motion. Each pulse is assumed to have the duration $\tau_{1}=\tau_{\mathrm{c}} / 2$, so that at resonance $(\eta=0)$ we have complete conversion. The dimensionless running variables are $\theta_{0}$ (waiting time in units of conversion time $\tau_{\mathrm{c}}$ ) and $\eta=\delta / 2 g$.

From the viewpoint of the experiment the most interesting case assumes the total interaction time of the quadrupole radiation to be exactly equal to the conversion time $\tau_{\mathrm{c}}$, that means $\theta_{1}=0.5$. Then for $\eta=0$ and any value of $\theta_{0}$ the profile function is $f_{2}\left(0 ; \theta_{0}, 0.5\right)=1$. Fig. 5 shows the landscape generated by $f_{2}\left(\eta ; \theta_{0}, \theta_{1}=0.5\right)$. Sections through this landscape at constant waiting time $\theta_{0}$ generate the line shape expected to be seen in experiment. Fig. 6 shows a typical line shape for two-pulse Ramsey excitation, calculated for a waiting time $\theta_{0}=1.5$ in comparison to the line shape for one-pulse excitation.

It is interesting to observe that by the use of the Ramsey method complete conversion of pure magnetron motion into modified cyclotron motion becomes possible even at off-resonance frequencies $(\delta \neq 0)$, as long as the condition $\eta^{2}=(\delta / 2 g)^{2} \leq 1$ is satisfied. In different words, pulse duration $\theta_{1}$ and waiting time $\theta_{0}$ can be chosen in such a way that a given sideband in the range $-1 \leq \eta \leq+1$ provides complete conversion. To obtain this result we determine the pulse duration $\theta_{1}$ so that the tip of the normalized Bloch vector (see Fig. 1) with the initial components $(0,0,-1)$ follows the heavily lined circular arc from the south pole all the way up to the equator. On the equator the 3rd component $T_{3}^{\prime}$ must vanish, thus the pulse duration is determined by the condition that the 33-element of the matrix (70) vanishes, $M_{33}=0$. In dimensionless variables this condition reads $\eta^{2}+\cos \left(\pi \theta_{1} \sqrt{1+\eta^{2}}\right)=0$, with the solution

$$
\theta_{1}=\frac{1}{2 \sqrt{1+\eta^{2}}}+\frac{\arcsin \left(\eta^{2}\right)}{\pi \sqrt{1+\eta^{2}}} \quad\left(\eta^{2} \leq 1\right)
$$

During the waiting period $\theta_{0}$ the tip of the Bloch vector moves along the equator until it intersects the upward directed branch of the other heavily lined circle (Fig. 1) that passes through the north pole, in addition it may eventually fully circle the equator several times. Finally during the second Ramsey pulse the tip of the Bloch vector moves along this upward directed circular arc to the north pole. The appropriate value of the waiting time follows from the condition for complete conversion, $f_{2}\left(\eta ; \theta_{0}, \theta_{1}\right)=1$. Using the

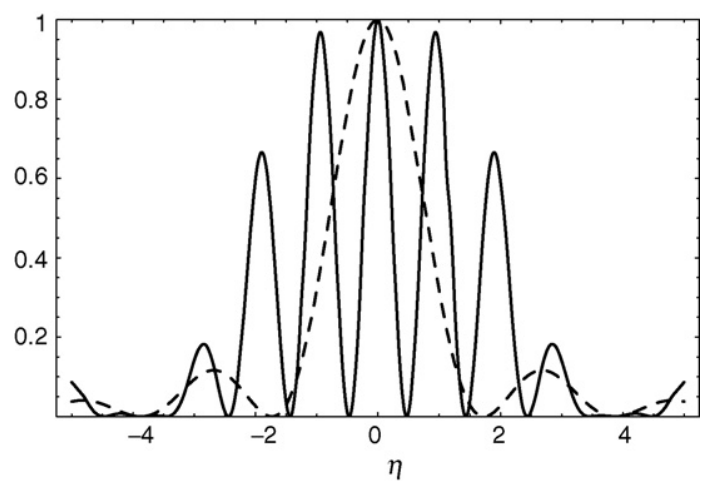

Fig. 6. A cross section of the landscape profile corresponding to a fixed value of $\theta_{0}$ yields the expected line shape for that particular value of the waiting time. The figure shows the line shape as a function of $\eta$ for $\theta_{0}=1.5$ (solid line) in comparison to the line shape for one-pulse excitation $\left(\theta_{0}=0\right.$, dashed line). 

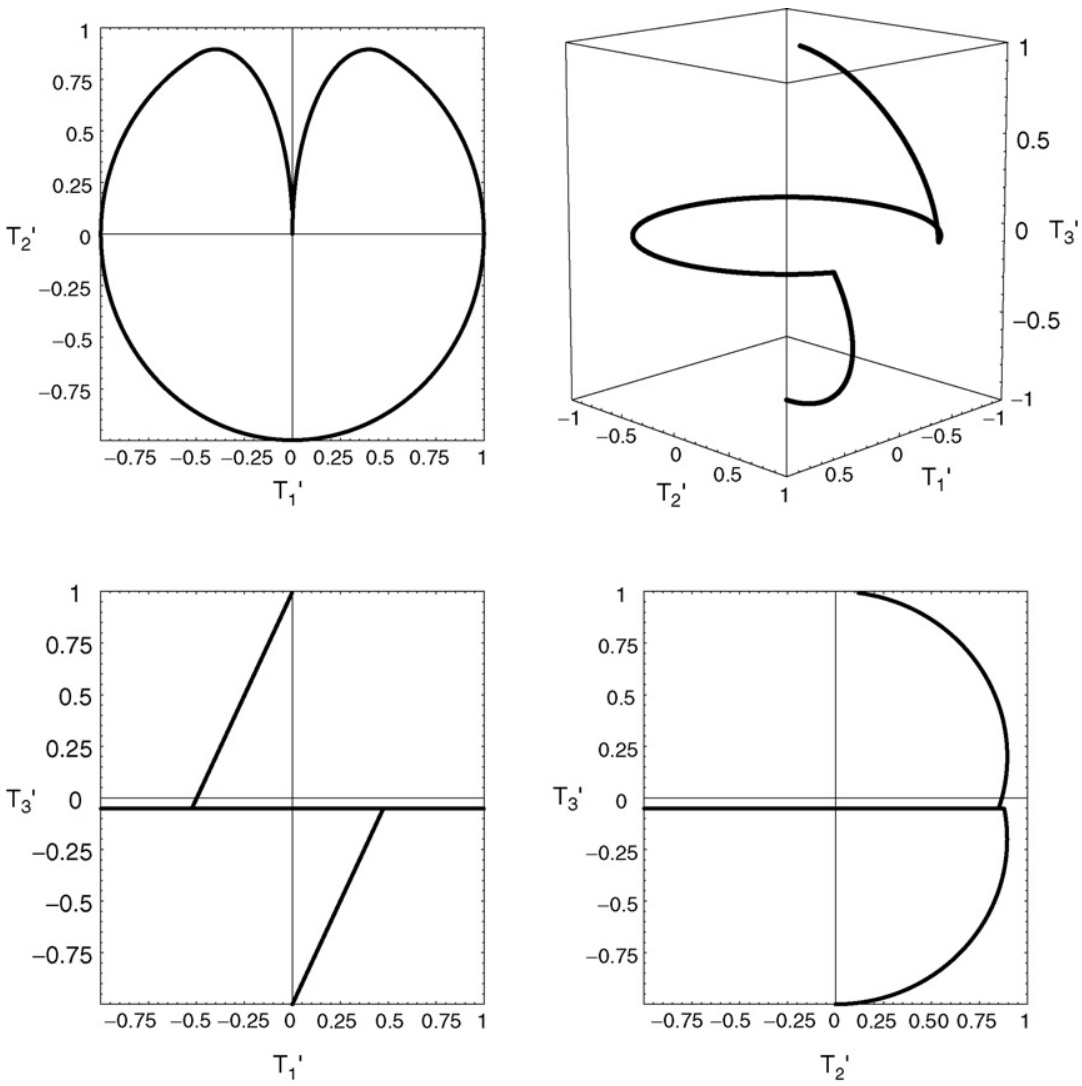

Fig. 7. Path described by the tip of the Bloch vector at the maximum of the first sideband, three-dimensional view and projections onto the $1^{\prime} 2^{\prime}$-plane, the $1^{\prime} 3^{\prime}$-plane, and the $2^{\prime} 3^{\prime}$-plane. For this figure a detuning parameter $\eta=0.5$ is assumed. Each Ramsey pulse has duration $\theta_{1}=0.5$ [Eq. (97) would require $\left.\theta_{1}=0.519\right]$, too short for the Bloch vector to reach the equator. During the waiting time $\theta_{0}=3.333$ [determined from Eq. (98)] the tip of the Bloch vector moves on a parallel south of the equator, then the second Ramsey pulse takes it to an end point close to the north pole. Since the standard Ramsey method assumes $\theta_{1}=0.5$, which is not the exact solution of Eq. (97), a small component of magnetron motion remains.

above result for $\theta_{1}$ one determines the waiting time to be

$$
\theta_{0}=\frac{2}{\eta}\left(n-\frac{1}{\pi} \arcsin (\eta)\right) \quad(n=1,2,3, \ldots)
$$

where the integer $n$ tells us that we have achieved complete conversion for the $n$th sideband.

The mechanism just described also explains qualitatively why with standard Ramsey excitation $\left(\theta_{1}=0.5\right)$ the sidebands nearest to the central peak are very prominent. The reason is that for the parameters of these sidebands the Eqs. (97) and (98) are approximately satisfied. As an illustration we analyze in Fig. 7 the path described by the tip of the Bloch vector at the maximum of the 1st sideband.

There are various ways to generalize these considerations. For example, previously we assumed the second pulse of quadrupole radiation to be exactly in phase with the first pulse. We might, however, apply this second pulse with a phaseshift $\chi$ relative to the first pulse. Then the calculation of the Ramsey matrix $\mathcal{R}^{(2)}\left(\delta ; \tau_{0}, \tau_{1}, g, \chi\right)$ requires a slight modification. On the basis of Eq. (42) the time development of the system is now described by

$$
\begin{aligned}
\left(\begin{array}{c}
a_{+}\left(t_{0}+\tau_{0}+2 \tau_{1}\right) \\
a_{-}\left(t_{0}+\tau_{0}+2 \tau_{1}\right)
\end{array}\right)= & \mathrm{e}^{-(i / 2)\left(\tau_{0}+2 \tau_{1}\right)} \mathcal{N}^{-1}\left(\phi_{\mathrm{d}}\left(t_{0}+\tau_{0}+2 \tau_{1}\right)+\chi\right) \cdot \mathcal{M}\left(\tau_{1} ; \delta, g\right) \cdot \mathcal{N}\left(\phi_{\mathrm{d}}\left(t_{0}+\tau_{0}+\tau_{1}\right)+\chi\right) \\
& \cdot \mathcal{N}^{-1}\left(\phi_{\mathrm{d}}\left(t_{0}+\tau_{0}+\tau_{1}\right)\right) \cdot \mathcal{M}\left(\tau_{1} ; \delta, 0\right) \cdot \mathcal{M}\left(\tau_{1} ; \delta, g\right) \cdot \mathcal{N} \phi_{\mathrm{d}}\left(t_{0}\right)\left(\begin{array}{l}
a_{+}\left(t_{0}\right) \\
a_{-}\left(t_{0}\right)
\end{array}\right) .
\end{aligned}
$$

From this relation we extract the complex $2 \times 2$ Ramsey matrix

$$
\mathcal{R}^{(2)}\left(\delta ; \tau_{0}, \tau_{1}, g, \chi\right)=\mathcal{M}\left(\tau_{1} ; \delta, g\right) \cdot\left(\begin{array}{cc}
\mathrm{e}^{(i / 2)\left(\delta \tau_{0}+\chi\right)} & 0 \\
0 & \mathrm{e}^{-(i / 2)\left(\delta \tau_{0}+\chi\right)}
\end{array}\right) \cdot \mathcal{M}\left(\tau_{1} ; \delta, g\right) .
$$




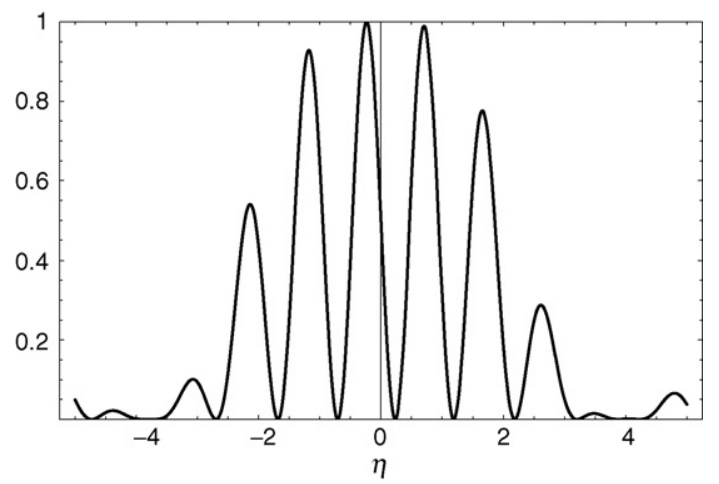

Fig. 8. line shape for two-pulse Ramsey excitation with a phaseshift of $\pi / 2$ for the second pulse. At the exact resonance frequency $(\eta=0)$ the dispersion type curve has the value 0.5 . The figure is calculated with $\theta_{0}=1.5$. With a phaseshift of $-\pi / 2$ the line shape is the mirror image at $\eta=0$ of the curve shown above.

Again the two-pulse profile function is obtained as $F_{2}\left(\delta ; \tau_{0}, \tau_{1}, g, \chi\right)=\left|\mathcal{R}_{12}^{(2)}\left(\delta ; \tau_{0}, \tau_{1}, g, \chi\right)\right|^{2}$, by expansion of this expression we find

$$
F_{2}\left(\delta ; \tau_{0}, \tau_{1}, g, \chi\right)=\frac{4 g^{2}}{\omega_{\mathrm{R}}^{2}}\left\{\cos \left(\frac{\delta \tau_{0}}{2}+\frac{\chi}{2}\right) \sin \left(\omega_{\mathrm{R}} \tau_{1}\right)+\frac{\delta}{\omega_{\mathrm{R}}} \sin \left(\frac{\delta \tau_{0}}{2}+\frac{\chi}{2}\right)\left[\cos \left(\omega_{\mathrm{R}} \tau_{1}\right)-1\right]\right\}^{2} .
$$

In his Nobel lecture [4] Ramsey draws attention to the fact that dispersion type line shapes provide maximum sensitivity for detecting small shifts in the resonance frequency. In fact, for the experimental setup considered in this paper an additional phase of $\pm \pi / 2$ for the second pulse of quadrupole radiation shifts the line pattern in such a way that the exact resonance frequency $(\eta=0)$ lies exactly in the middle of the steep slope of the central Ramsey fringe, see Fig. 8.

It is of interest to relate the two-pulse profile function with phaseshift to our previous discussion of the Fourier transform of a sequence of two wave trains of a scalar wave, Eq. (85). As in the case of one-pulse excitation the substitution $\omega_{\mathrm{R}}=\sqrt{\delta^{2}+4 g^{2}} \rightarrow \delta$ (eqivalent to $\sqrt{1+\eta^{2}} \rightarrow \eta$ ) transforms the two-pulse profile function Eq. (101) into the Fourier transform of a two-pulse wave train Eq. (85). Thus we can conclude that aside from fine details of the line shape the gross structure of expression (101) is determined by the Fourier transformation.

For yet another generalization we admit that the two Ramsey pulses may be of unequal duration $\tau_{1}, \tau_{2}$. The same kind of reasoning as before results in the generalized two-pulse profile function

$$
\begin{aligned}
F_{2}\left(\delta ; \tau_{0}, \tau_{1}, \tau_{2}, g\right)= & +\frac{4 g^{2}}{\omega_{\mathrm{R}}^{2}}\left\{\cos \left(\delta \tau_{0} / 2\right) \sin \left(\omega_{\mathrm{R}}\left(\tau_{1}+\tau_{2}\right) / 2\right)+\frac{\delta}{\omega_{\mathrm{R}}} \sin \left(\delta \tau_{0} / 2\right)\left[\cos \left(\omega_{\mathrm{R}}\left(\tau_{1}+\tau_{2}\right) / 2\right)-\cos \left(\omega_{\mathrm{R}}\left(\tau_{1}-\tau_{2}\right) / 2\right)\right]\right\}^{2} \\
& +\frac{4 g^{2}}{\omega_{\mathrm{R}}^{2}} \cdot \sin ^{2}\left(\delta \tau_{0} / 2\right) \cdot \sin ^{2}\left(\omega_{\mathrm{R}}\left(\tau_{1}-\tau_{2}\right) / 2\right) .
\end{aligned}
$$

For $\tau_{2}=0$ this expression reduces to the one-pulse profile function $F_{1}\left(\delta ; \tau_{1}, g\right)$. Keeping $\tau_{1}+\tau_{2}=\tau_{\text {tot }}$ constant, the smallest width for the central Ramsey fringe is obtained for the symmetric situation $\tau_{1}=\tau_{2}=\tau_{\text {tot }} / 2$, which therefore appears to be experimentally the most interesting one.

\subsubsection{Ramsey excitation in terms of classical trajectories}

The study of classical ion trajectories can very much support our intuition about the mechanism of Ramsey excitation. Basically one is interested to follow the trajectory of an ion with given initial position $x_{0}=x\left(t_{0}\right), y_{0}=y\left(t_{0}\right)$ and given initial velocity $v_{x 0}=\dot{x}\left(t_{0}\right), v_{y 0}=\dot{y}\left(t_{0}\right)$ through the various stages of the Ramsey excitation procedure. As described in a preceding section, the operators $x, y, \dot{x}$, and $\dot{y}$ are related to the annihilation and creation operators $a_{ \pm}$and $a_{ \pm}^{\dagger}$ by Eqs. (16), (17), (19), and (20). Taking the expectation value of these equations for a coherent state $\left|\alpha_{+}(0), \alpha_{-}(0)\right\rangle$, using Eq. (46), one obtains the initial data of the trajectory in terms of the initial complex amplitudes $\alpha_{+}(0)$ and $\alpha_{-}(0)$ of the magnetron and modified cyclotron harmonic oscillators. Inverting these equations we find

$$
\begin{aligned}
& \alpha_{+}(0)=\sqrt{\frac{m}{2 \hbar \omega_{1}}} \cdot\left[-\left(v_{y 0}+\omega_{-} x_{0}\right)+i\left(v_{x 0}-\omega_{-} y_{0}\right)\right], \\
& \alpha_{-}(0)=\sqrt{\frac{m}{2 \hbar \omega_{1}}} \cdot\left[+\left(v_{y 0}+\omega_{+} x_{0}\right)+i\left(v_{x 0}-\omega_{+} y_{0}\right)\right] .
\end{aligned}
$$



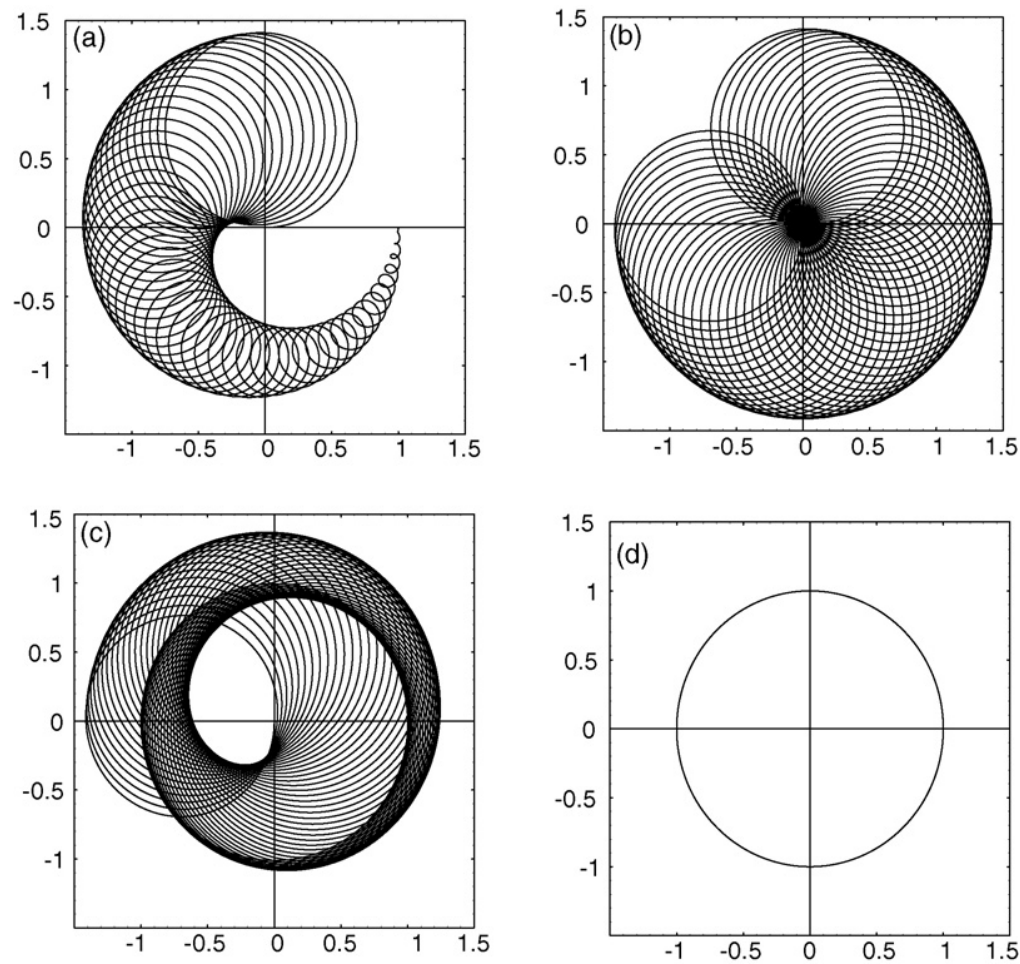

Fig. 9. Ion trajectory during two-pulse Ramsey excitation when we have exact resonance $(\delta=0)$. (a) First Ramsey pulse $0 \leq t \leq \tau_{\mathrm{c}} / 2$ : The trajectory starts from a circular pure magnetron orbit of radius 1 , the magnetron radius $R_{-}(t)$ decreases from 1 to $\sqrt{0.5}$, while at the same time the modified cyclotron radius $R_{+}(t)$ increases from 0 to $\sqrt{0.5}$. (b) Waiting time $\tau_{\mathrm{c}} / 2 \leq t \leq \tau_{\mathrm{c}} / 2+\tau_{0}$ : The ion follows a rosette type orbit. The center of the fast circular cyclotron motion of radius $R_{+}=\sqrt{0.5}$ moves itself on a circle of radius $R_{-}=\sqrt{0.5}$ at a slow speed which is determined by the magnetron frequency. All cyclotron circles pass through the origin. (c) Second Ramsey pulse $\tau_{\mathrm{c}} / 2+\tau_{0} \leq t \leq \tau_{\mathrm{c}}+\tau_{0}$ : the modified cyclotron radius $R_{+}(t)$ continues to increase from $\sqrt{0.5}$ to 1 , while at the same time the magnetron radius $R_{-}(t)$ is decreasing from $\sqrt{0.5}$ to 0 . (d) After the two-pulse Ramsey excitation is completed the ion moves on a pure cyclotron orbit of radius $R_{+}=1$.

The corresponding initial values of the modified cyclotron radius $R_{+}(0)$ and the magnetron radius $R_{-}(0)$ follow from Eq. (49). The initial complex amplitudes $\alpha_{+}(0)$ and $\alpha_{-}(0)$ can now be inserted into the Eq. (54) and the development of the ion trajectory under quadrupole excitation can be calculated. We are especially interested in the initial state of pure magnetron motion, $\alpha_{+}(0)=0$. For a trajectory starting at the point with coordinates $x_{0}, y_{0}$ this condition implies the initial velocity $v_{x 0}=\omega_{-} y_{0}, v_{y 0}=-\omega_{-} x_{0}$, yielding $\alpha_{-}(0)=\sqrt{m \omega_{1} /(2 \hbar)}\left(x_{0}-i y_{0}\right)$. Fig. 9 shows the ion trajectory with starting point $x_{0}=1, y_{0}=0$ as it develops during a full Ramsey excitation cycle, assuming that the driving quadrupole field is exactly in resonance $(\delta=0)$.

For slightly different initial velocities $v_{x 0}=\omega_{-} y_{0}+\delta v_{x 0}, v_{y 0}=-\omega_{-} x_{0}+\delta v_{y 0}$ a small amount of modified cyclotron motion is present from the beginning, with a modified cyclotron radius $R_{+}(0)=\omega_{1}^{-1} \sqrt{\delta v_{x 0}^{2}+\delta v_{y 0}^{2}}$. A representative example is illustrated in Fig. 10.

\subsection{More general excitation schemes}

A systematic exploration of the advantages offered by Ramsey's method of separated oscillating fields would be incomplete without a first look at more complicated excitation schemes using three or more pulses of quadrupole radiation, even though useful applications of such excitation schemes in the field of high-accuracy mass spectrometry may not yet be in sight. We restrict our discussion to the simplest and most symmetric schemes using three, four, or five pulses of equal duration $\tau_{1}$ and equal waiting times $\tau_{0}$ between the pulses. The procedure for deriving the $n$-pulse profile function $F_{n}\left(\delta ; \tau_{0}, \tau_{1}, g\right)$ follows the line of arguments in the preceding subsection. Thus we start with the statement

$$
F_{n}\left(\delta ; \tau_{0}, \tau_{1}, g\right)=\left\langle N_{+}\left(t_{0}+(n-1) \tau_{0}+n \tau_{1}\right)\right\rangle /\left\langle N_{\text {tot }}\right\rangle=\frac{1}{2}\left[1+\left\langle T_{3}\left(t_{0}+(n-1) \tau_{0}+n \tau_{1}\right)\right\rangle /\left\langle T_{0}\right\rangle\right] .
$$

In order to calculate $\left\langle T_{3}\left(t_{2 n-1}\right)\right\rangle$, where for brevity we have written $t_{2 n-1}=t_{0}+(n-1) \tau_{0}+n \tau_{1}$, we use again our general solution for the time development of the Bloch vector operator, Eq. (72). Similar reasoning as above yields the general result

$$
\left(\begin{array}{c}
\left\langle T_{1}\left(t_{2 n-1}\right)\right\rangle \\
\left\langle T_{2}\left(t_{2 n-1}\right)\right\rangle \\
\left\langle T_{3}\left(t_{2 n-1}\right)\right\rangle
\end{array}\right)=\mathrm{N}^{-1}\left(\phi_{\mathrm{d}}\left(t_{2 n-1}\right)\right) \cdot \mathrm{R}^{(n)}\left(\delta ; \tau_{0}, \tau_{1}, g\right) \cdot \mathrm{N}\left(\phi_{\mathrm{d}}\left(t_{0}\right)\right)\left(\begin{array}{c}
\left\langle T_{1}\left(t_{0}\right)\right\rangle \\
\left\langle T_{2}\left(t_{0}\right)\right\rangle \\
\left\langle T_{3}\left(t_{0}\right)\right\rangle
\end{array}\right)
$$



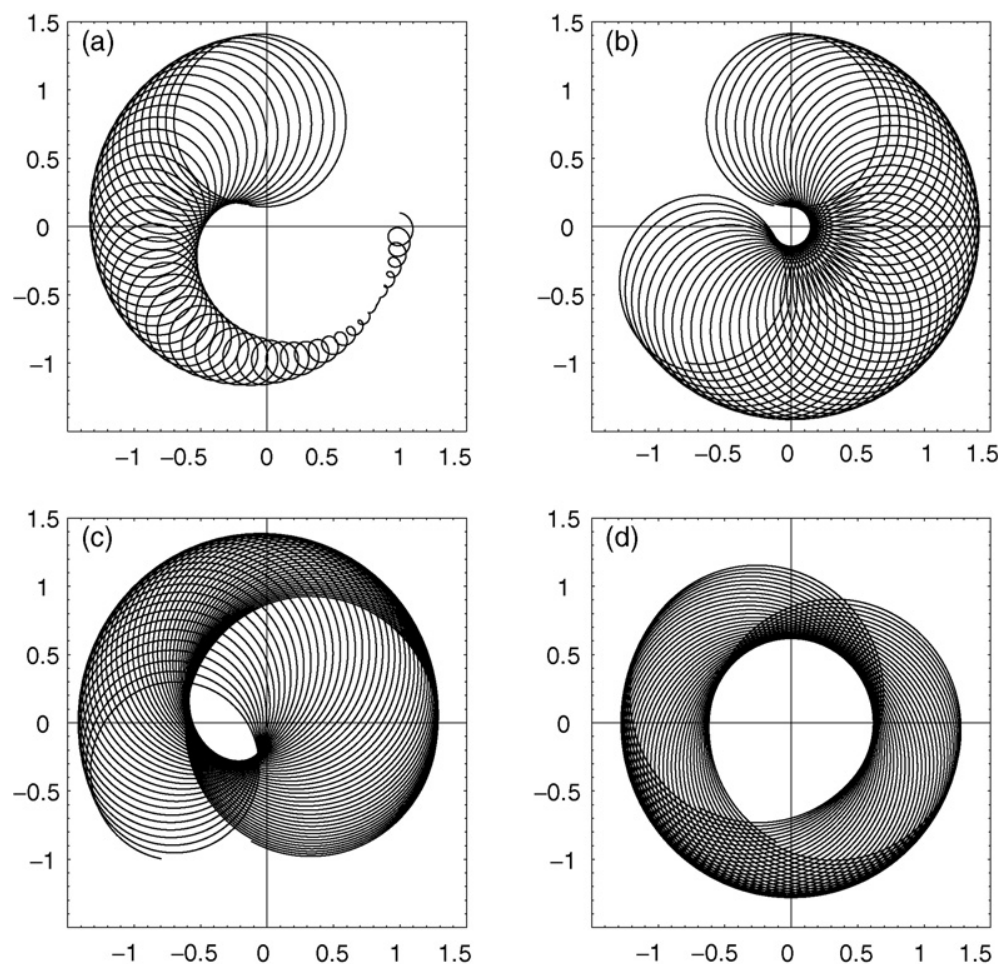

Fig. 10. Ion trajectory during two-pulse Ramsey excitation with detuning $(\eta=0.5)$ and initially a small component of modified cyclotron motion. (a) First Ramsey pulse $0 \leq t \leq \tau_{\mathrm{c}} / 2$ : Due to our choice of phases the small cyclotron component is first converted to magnetron motion, then magnetron motion is converted to modified cyclotron motion. The conversion result at the end of the first Ramsey pulse is described by the inequality $R_{+}\left(\tau_{\mathrm{c}} / 2\right)<\sqrt{0.5}<R_{-}\left(\tau_{\mathrm{c}} / 2\right)$. (b) Waiting time $\tau_{\mathrm{c}} / 2 \leq t \leq \tau_{\mathrm{c}} / 2+\tau_{0}$ : The radii $R_{+}$and $R_{-}$remain constant, the ion trajectory is located in a ring-shaped region with outer radius $R_{+}\left(\tau_{\mathrm{c}} / 2\right)+R_{-}\left(\tau_{\mathrm{c}} / 2\right)$ and inner radius $R_{-}\left(\tau_{\mathrm{c}} / 2\right)-R_{+}\left(\tau_{\mathrm{c}} / 2\right)$. (c) Second Ramsey pulse $\tau_{\mathrm{c}} / 2+\tau_{0} \leq t \leq \tau_{\mathrm{c}}+\tau_{0}$ : The conversion of magnetron into modified cyclotron motion continues, but remains incomplete at the end of the second Ramsey pulse. (d) The Ramsey excitation procedure has left over a certain amount of magnetron motion, so that $R_{-}\left(\tau_{\mathrm{c}}+\tau_{0}\right)<R_{+}\left(\tau_{\mathrm{c}}+\tau_{0}\right)$. Thus the center of a large cyclotron circle drifts along a magnetron circle of smaller radius.

with the $n$-pulse Ramsey matrix

$$
\mathrm{R}^{(n)}\left(\delta ; \tau_{0}, \tau_{1}, g\right)=\mathrm{M}\left(\tau_{1} ; \delta, g\right) \cdot\left[\mathrm{M}\left(\tau_{0} ; \delta, 0\right) \cdot \mathrm{M}\left(\tau_{1} ; \delta, g\right)\right]^{n-1} .
$$

Finally taking into account the initial condition $\left\langle T_{1}\left(t_{0}\right)\right\rangle=\left\langle T_{2}\left(t_{0}\right)\right\rangle=0$ and the fact that the calculation of $F_{n}$ requires only the component $T_{3}$, which is not affected by $\mathrm{N}\left(\phi_{\mathrm{d}}(t)\right)$, we arrive at

$$
F_{n}\left(\delta ; \tau_{0}, \tau_{1}, g\right)=\frac{1}{2}\left[1+\left\langle T_{3}\left(t_{2 n-1}\right)\right\rangle /\left\langle T_{0}\right\rangle\right]=\frac{1}{2}\left(1-\mathrm{R}_{33}^{(n)}\left(\delta ; \tau_{0}, \tau_{1}, g\right)\right) .
$$

The evaluation of this expression requires cumbersome algebra. More convenient is the alternative approach of arguing that

$$
\left\langle N_{+}\left(t_{2 n-1}\right)\right\rangle=\| a_{+}\left(t_{2 n-1}\right)|\psi\rangle \|^{2}=\left|\mathcal{R}_{12}^{(n)}\left(\delta ; \tau_{0}, \tau_{1}, g\right)\right|^{2} \cdot\left\langle N_{-}\left(t_{0}\right)\right\rangle,
$$

where $\mathcal{R}_{12}^{(n)}$ denotes the 12-element of the complex $2 \times 2 n$-pulse Ramsey matrix

$$
\mathcal{R}^{(n)}\left(\delta ; \tau_{0}, \tau_{1}, g\right)=\mathcal{M}\left(\tau_{1} ; \delta, g\right) \cdot\left[\mathcal{M}\left(\tau_{0} ; \delta, 0\right) \cdot \mathcal{M}\left(\tau_{1} ; \delta, g\right)\right]^{n-1} .
$$

The $n$-pulse profile function is then represented as the absolute square of a complex quantity,

$$
F_{n}\left(\delta ; \tau_{0}, \tau_{1}, g\right)=\left\langle N_{+}\left(t_{2 n-1}\right)\right\rangle /\left\langle N_{\text {tot }}\right\rangle=\left|\mathcal{R}_{12}^{(n)}\left(\delta ; \tau_{0}, \tau_{1}, g\right)\right|^{2} .
$$

This expression has been evaluated explicitely for $n=3,4,5$, the results are quoted below using the dimensionless variables $\eta=\delta / 2 g, \theta_{0}=\tau_{0} / \tau_{\mathrm{c}}$, and $\theta_{1}=\tau_{1} / \tau_{\mathrm{c}}$. To reduce the formulas to a manageable size the following abbreviations are used,

$$
C=C\left(\eta, \theta_{1}\right)=\cos \left(\frac{\pi}{2} \theta_{1} \sqrt{1+\eta^{2}}\right), \quad S=S\left(\eta, \theta_{1}\right)=\sin \left(\frac{\pi}{2} \theta_{1} \sqrt{1+\eta^{2}}\right) .
$$

For completeness the one- and two-pulse profile functions are also listed in this notation.

$$
f_{1}\left(\eta ; \theta_{1}\right)=\frac{1}{1+\eta^{2}} \cdot S^{2}
$$




$$
\begin{aligned}
& f_{2}\left(\eta ; \theta_{0}, \theta_{1}\right)=\frac{4}{1+\eta^{2}} \cdot S^{2} \cdot\left\{C \cdot \cos \left(\frac{\pi}{2} \theta_{0} \eta\right)-\frac{\eta}{\sqrt{1+\eta^{2}}} \cdot S \cdot \sin \left(\frac{\pi}{2} \theta_{0} \eta\right)\right\}^{2} \\
& f_{3}\left(\eta ; \theta_{0}, \theta_{1}\right)=\frac{4}{1+\eta^{2}} \cdot S^{2} \cdot\left\{\left(C^{2}-\frac{\eta^{2}}{1+\eta^{2}} \cdot S^{2}\right) \cos \left(\pi \theta_{0} \eta\right)-\frac{2 \eta}{\sqrt{1+\eta^{2}}} \cdot C \cdot S \cdot \sin \left(\pi \theta_{0} \eta\right)+\frac{1}{2}-\frac{1}{1+\eta^{2}} \cdot S^{2}\right\}^{2} \\
& f_{4}\left(\eta ; \theta_{0}, \theta_{1}\right)=\frac{4}{1+\eta^{2}} \cdot S^{2} \cdot\left\{\left(C^{3}-\frac{3 \eta^{2}}{1+\eta^{2}} \cdot C \cdot S^{2}\right) \cos \left(\frac{3 \pi}{2} \theta_{0} \eta\right)-\left(\frac{3 \eta}{\sqrt{1+\eta^{2}}} \cdot C^{2} \cdot S-\frac{\eta^{3}}{{\sqrt{1+\eta^{2}}}^{3}} \cdot S^{3}\right)\right. \\
& \left.\times \sin \left(\frac{3 \pi}{2} \theta_{0} \eta\right)+\left[C \cdot \cos \left(\frac{\pi}{2} \theta_{0} \eta\right)-\frac{\eta}{\sqrt{1+\eta^{2}}} \cdot S \cdot \sin \left(\frac{\pi}{2} \theta_{0} \eta\right)\right] \cdot\left[1-\frac{3}{1+\eta^{2}} \cdot S^{2}\right]\right\}^{2} \\
& f_{5}\left(\eta ; \theta_{0}, \theta_{1}\right)=\frac{4}{1+\eta^{2}} \cdot S^{2} \cdot\left\{\left(C^{4}-\frac{6 \eta^{2}}{1+\eta^{2}} \cdot C^{2} \cdot S^{2}+\frac{\eta^{4}}{\left(1+\eta^{2}\right)^{2}} \cdot S^{4}\right) \cos \left(2 \pi \theta_{0} \eta\right)\right. \\
& -\left(\frac{4 \eta}{\sqrt{1+\eta^{2}}} \cdot C^{3} \cdot S-\frac{4 \eta^{3}}{{\sqrt{1+\eta^{2}}}^{3}} \cdot C \cdot S^{3}\right) \sin \left(2 \pi \theta_{0} \eta\right) \\
& +\left[\left(C^{2}-\frac{\eta^{2}}{1+\eta^{2}} \cdot S^{2}\right) \cos \left(\pi \theta_{0} \eta\right)-\frac{2 \eta}{\sqrt{1+\eta^{2}}} \cdot C \cdot S \cdot \sin \left(\pi \theta_{0} \eta\right)\right] \cdot\left[1-\frac{4}{1+\eta^{2}} \cdot S^{2}\right] \\
& \left.+\frac{1}{2}-\frac{3}{1+\eta^{2}} \cdot S^{2}+\frac{3}{\left(1+\eta^{2}\right)^{2}} \cdot S^{4}\right\}^{2}
\end{aligned}
$$

These profile functions depend on the three variables $\eta, \theta_{0}$, and $\theta_{1}$. For a visualization by a three-dimensional surface we must keep one of them fixed. From the experimental viewpoint the most interesting choice is $n \tau_{1}=\tau_{\mathrm{c}}$, in which case the total duration of all $n$ pulses equals the conversion time $\tau_{\mathrm{c}}$ at resonance. This choice translates into the dimensionless variables as $\theta_{1}=n^{-1}$. As a representative example we display in Fig. 11 the profile landscape for five-pulse excitation. Sections through the landscape at constant $\tau_{0}$ provide the desired line shapes as a function of $\eta$. Fig. 12 shows the line shapes for two-, three-, four-, and five-pulse excitation, assuming the total excitation time $n \tau_{1}=\tau_{\mathrm{c}}$. Wheras for $n=2$ the pattern consisted of a central peak with large satellite peaks, for larger $n$ many of the satellite peaks shrink into little ripples. The valleys between large peaks become wider, showing $n-2$ ripples at the bottom.

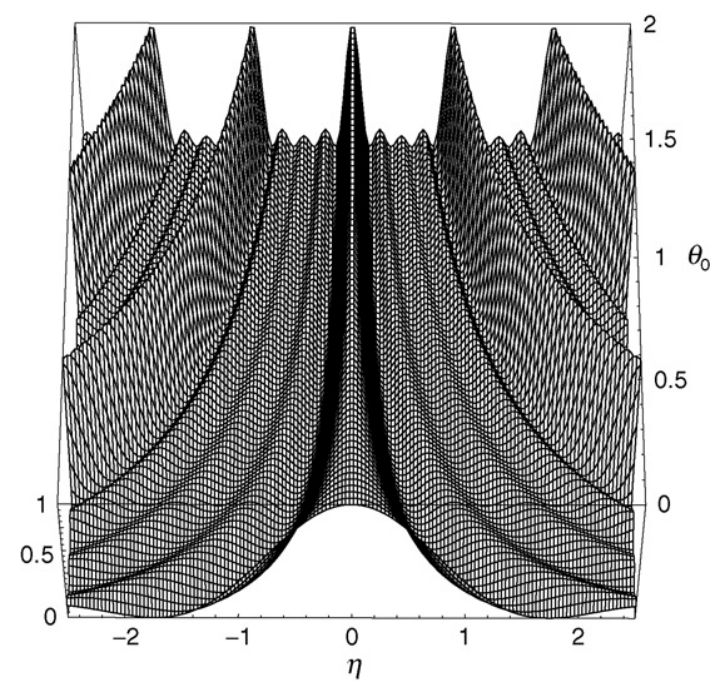

Fig. 11. Landscape profile for five-pulse Ramsey excitation showing the probability for the conversion of pure magnetron motion into modified cyclotron motion. Each pulse is assumed to have the duration $\tau_{1}=\tau_{\mathrm{c}} / 5$, so that at resonance $(\eta=0)$ we have complete conversion. The dimensionless running variables are $\theta_{0}$ (waiting time in units of conversion time $\tau_{\mathrm{c}}$ ) and $\eta=\delta / 2 g$. 

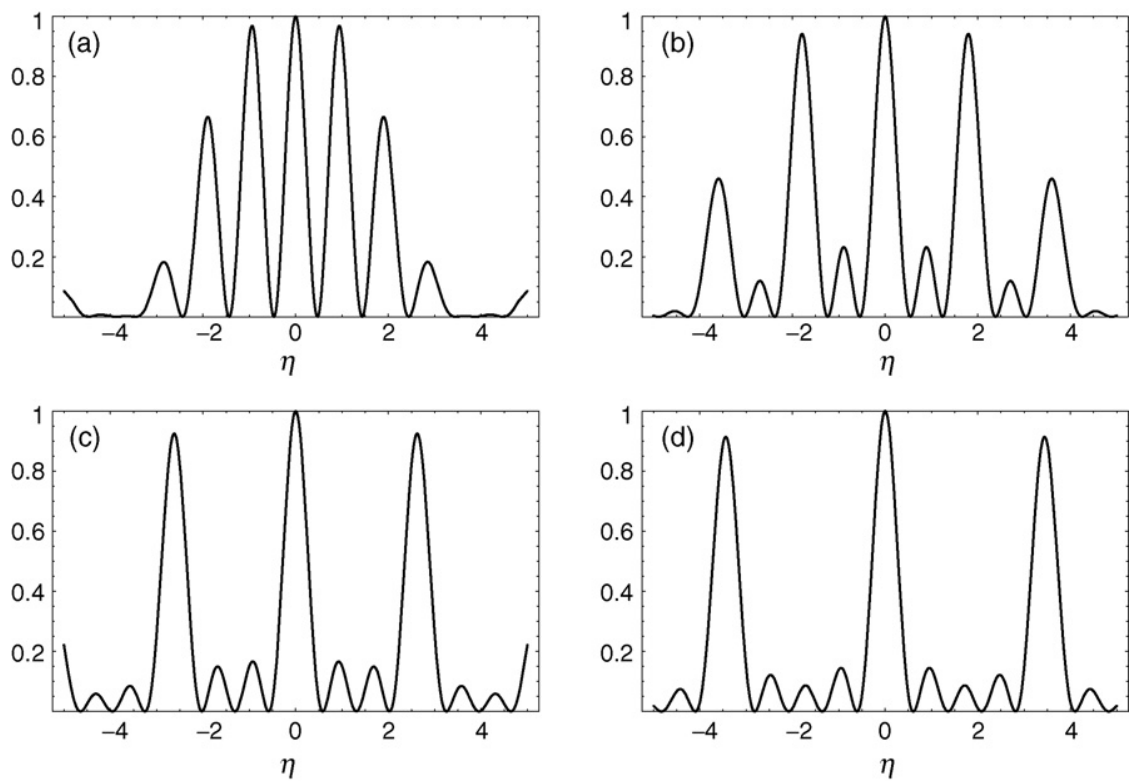

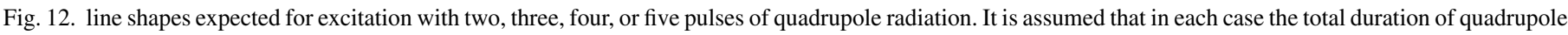

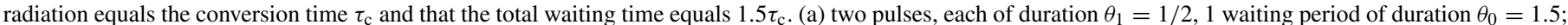

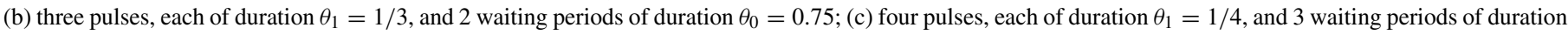
$\theta_{0}=0.5$; (d) five pulses, each of duration $\theta_{1}=1 / 5$, and 4 waiting periods of duration $\theta_{0}=0.375$.

\section{Concluding remarks}

The work described in this paper on the one hand intends to clarify conceptual problems connected with the application of Ramsey's method of separated oscillating fields to the physics of ion motion in a Penning trap, on the other hand it provides a detailed theory of the resonance line shapes, intended for application in high-accuracy mass spectrometry. To start with we required a simple and exactly solvable model for the interaction of an external rf-quadrupole field with an ion confined in an ideal Penning trap. Here the viewpoint of quantum theory was most helpful. The essence of the interaction is the absorption of a photon of the driving quadrupole field with frequency $\omega_{\mathrm{d}} \approx \omega_{\mathrm{c}}$ with simultaneous creation of a quantum of modified cyclotron energy $\hbar \omega_{+}$and annihilation of a quantum of (negative) magnetron energy $\hbar \omega_{-}$, together with the inverse process. An important feature of this interaction is that the total number of oscillator quanta in the system remains constant. As a consequence the expectation value of the Bloch vector, i.e., a vector operator constructed from the creation and annihilation operators of the modified cyclotron and magnetron oscillators and possessing the commutation rules of an angular momentum, is a conserved three-vector that performs precessional and nutational motions which can be visualized on the surface of a sphere. By means of this concept a close analogy to nuclear-magnetic-resonance can be established. This appears to be a very valuable insight. For the application to precision mass spectrometry the crucial step is the resonant conversion of magnetron motion into modified cyclotron motion for the ions caught in the precision trap. The probability of this conversion process is determined by the 3 -component of the Bloch vector $T_{3}$. Therefore, in order to gain information on the conversion process we study the dependence of the Bloch vector on the duration and structure of the pulses of the external rf-quadrupole radiation, in particular we study the dependence on the detuning in order to learn about the line shapes. In the Ramsey technique the entire amount of rf-quadrupole radiation that is necessary at the resonance frequency $\omega_{\mathrm{c}}$ for a complete conversion of the magnetron mode into the modified cyclotron mode is applied in two or more pulses with waiting times in between. The theoretically expected line shapes have been calculated for two-, three-, four-, and five-pulse conversion. The central peak is found to become narrower with increasing waiting time, we interpret this as a consequence of the time-energy uncertainty relation. Finally we ask, why does Ramsey's method work at all? There are no interfering waves of any kind, only several pulses of coherent radiation applied at distinct, non-overlapping time intervals. The answer is that during the waiting times an important phase is changing proportional to the detuning and to the waiting time. The phase gain $\delta \tau_{0}$ brings about the dramatic narrowing of the resonance lines.

\section{Acknowledgement}

It is a pleasure to thank my colleagues Klaus Blaum, Sebastian George and Lutz Schweikhard for stimulating my interest in the Ramsey method, and for many fruitful and illuminating discussions on the experimental applications. 


\section{References}

[1] S. George, K. Blaum, F. Herfurth, A. Herlert, M. Kretzschmar, S. Nagy, S. Schwarz, L. Schweikhard, C. Yazidjian, IJMS 264 (2007) 110.

[2] N.F. Ramsey, Phys. Rev. 76 (1949) 996.

[3] N.F. Ramsey, Phys. Rev. 78 (1950) 695.

[4] N.F. Ramsey, Rev. Mod. Phys. 62 (1990) 541.

[5] G. Bollen, H.-J. Kluge, T. Otto, G. Savard, H. Stolzenberg, Nucl. Instrum. Meth. B70 (1992) 490.

[6] I. Bergström, C. Carlberg, T. Fritioff, G. Douysset, J. Schönfelder, R. Schuch, Nucl. Instrum. Meth. A487 (2002) 618.

[7] G. Gräff, H. Kalinowsky, J. Traut, Z. Phys. A297 (1980) 35.

[8] M. Kretzschmar, in: D.H.E. Dubin, D. Schneider (Eds.), Trapped Charged Particles and Fundamental Physics, AIP Conf. Proc. vol. 457, The American Institute of Physics, 1999, p. 242.

[9] L.S. Brown, G. Gabrielse, Rev. Mod. Phys. 58 (1986) 233.

[10] K. Blaum, Phys. Rep. 425 (2006) 1.

[11] K. Pradip, Ghosh, Ion Traps, Clarendon Press, Oxford, 1995.

[12] F.G. Major, V.N. Gheorghe, G. Werth, Charged Particle Traps, Springer-Verlag, Berlin, Heidelberg, 2005.

[13] M. Kretzschmar, Eur. J. Phys. 12 (1991) 240.

[14] M. Kretzschmar, Phys. Scr. 46 (1992) 544.

[15] G. Bollen, R.B. Moore, G. Savard, H. Stolzenberg, J. Appl. Phys. 68 (1990) 4355.

[16] L. Schweikhard, A.G. Marshall, J. Am. Soc. Mass Spectrom. 4 (1993) 433.

[17] M.O. Scully, M.S. Zubairy, Quantum Optics, Cambridge University Press, 1997.

[18] E. Schrödinger, Naturwissenschaften 14 (1926) 664.

[19] G. Shenheng, A.G. Marshall, J. Chem. Phys. 98 (1993) 4486.

[20] M. König, G. Bollen, H.-J. Kluge, T. Otto, J. Szerypo, Int. J. Mass Spectrom. 142 (1995) 116. 